\title{
Recurrent Ischemia in the Canine Heart Causes Recurrent Bursts of Free Radical Production That Have a Cumulative Effect on Contractile Function
} A Pathophysiological Basis for Chronic Myocardial "Stunning"

\author{
Roberto Bolli, *‡ Marcel Zughaib, * Xiao-Ying Li, * Xian-Liang Tang, *‡ Jian-Zhong Sun, J. Fernando Triana, * \\ and Paul B. McCay $\$$ \\ With the technical assistance of Gemma Wallis $\$$ \\ * Experimental Research Laboratory, Section of Cardiology, Baylor College of Medicine, Houston, Texas 77030; ${ }^{\ddagger}$ Experimental \\ Research Laboratory, Division of Cardiology, University of Louisville, Louisville, Kentucky 40292; and ${ }^{\S}$ Molecular Toxicology Research \\ Group, Oklahoma Medical Research Foundation, Oklahoma City, Oklahoma 73104
}

\begin{abstract}
Open-chest dogs (total number used, 117) underwent 105 min coronary occlusions $(O)$ interspersed with 10 min of reperfusion $(R)$. When systolic thickening fraction was measured 9 min after each $R$, the first $O-R$ cycle was found to cause the largest decrement, with only a slight additional loss during the next four cycles and no further loss during the last five cycles (group IV), suggesting that the first few episodes of ischemia preconditioned the myocardium against the stunning induced by the last five episodes. However, different results were obtained when the total deficit of wall thickening during the final 4-h $R$ interval was measured. The total deficit was similar after one and three 5min $O$ (groups V and VI, respectively), indicating that the first ischemic episode did precondition against the next two episodes; however, it was $\sim 2.5$-fold greater after $10 \mathrm{O}$ (group IV) than after 3, indicating that the first 3 episodes failed to precondition against the next 7 . Thus, at some point between the 4th and 10th 0 , the preconditioning effect was lost and recurrent ischemic episodes started to have a cumulative effect. Measurements of free radicals with $\alpha$-phenyl $N$-tert-butyl nitrone (PBN) demonstrated a burst of free radical generation immediately after the 1st, 5th, and 10th $R$ (group VIII). The total cumulative release of PBN adducts during the initial 5 min of reflow was $58 \%$ less after the 5th $R$ than after the 1 st $(P<0.05)$ but did not differ significantly between the 1st and 10th $R$. When administered throughout the 10 O-R cycles, the $\cdot O H$ scavenger mercaptopropionyl glycine significantly enhanced the recovery of function (group I) and markedly suppressed the formation of free radicals (group VII). However, the beneficial effects of mercaptopropionyl glycine were completely, or largely, lost if the drug was discontinued after the first five (group II) or eight (group III) O-R cycles, respectively, implying that $(a)$ the oxidative stress associated with the last five, or
\end{abstract}

Address correspondence to Roberto Bolli, M.D., Division of Cardiology, University of Louisville, Louisville, KY 40292. Phone: 502-852-1837; FAX: 502-852-6474.

Received for publication 25 October 1994 and accepted in revised form 3 May 1995.

J. Clin. Invest.

(C) The American Society for Clinical Investigation, Inc.

0021-9738/95/08/1066/19 \$2.00

Volume 96, August 1995, 1066-1084 even two, cycles was sufficient to cause severe postischemic dysfunction, and $(b)$ the cumulative injury caused by repetitive ischemic episodes is mediated by recurrent oxidative stress. This study provides direct in vivo evidence that oxygen radicals play an important role in the pathogenesis of myocardial stunning after repetitive ischemia, and implicates $\cdot O H$ as a primary culprit. Taken together, the data indicate that recurrent brief ischemic episodes result in recurrent bouts of oxyradical-mediated injury that have a cumulative effect on contractility, a situation that could lead to protracted or even chronic myocardial stunning. (J. Clin. Invest. 1995. 96:1066-1084.) Key words: postischemic myocardial dysfunction - mercaptopropionyl glycine $\bullet$ spin trapping $\cdot \boldsymbol{\alpha}$-phenyl $N$-tert-butyl nitrone $\bullet$ preconditioning

\section{Introduction}

It is well established that a single, brief episode of myocardial ischemia causes prolonged depression of contractile function $(1,2)$, or "stunning"' $(3)$, in experimental animals. The clinical situation, however, is frequently different from the experimental model of a single coronary occlusion because many patients experience multiple recurrent episodes of myocardial ischemia (painful or painless) in the same territory as a consequence of recurrent spasm and/or thrombosis $(4,5)$. Therefore, understanding the pathogenesis and pathophysiology of myocardial stunning after repetitive ischemia is of considerable clinical importance.

Studies in open-chest dogs (6-10) have demonstrated that repeated (10-16 occlusions) brief ( $5 \mathrm{~min}$ ) coronary occlusions result in prolonged depression of contractile performance despite the absence of irreversible damage. This model of myocardial stunning differs from the single 15-min occlusion model in several respects: the mechanical dysfunction develops gradually and its severity is unrelated to collateral flow, the duration of each ischemic episode is much shorter ( $5 \mathrm{vs.} 15 \mathrm{~min}$ ), and the total ischemic burden is much greater ( $50-80$ vs. $15 \mathrm{~min}$ ) (6$10)$. Furthermore, the exposure of the myocardium to brief bouts of ischemia has been postulated to induce adaptations that modify the outcome of the next ischemic bouts (10), a situation that does not exist in single occlusion models. In view of these important differences, concepts derived from one model may not necessarily be applicable to the other (11). At present, relatively less is known regarding the pathogenesis and pathophysiology of myocardial stunning after multiple ischemic episodes than after a single ischemic episode. Although a recent study (12) suggests that oxygen radicals contribute to postisch- 
emic dysfunction after repeated brief coronary occlusions, several issues remain unresolved and need to be addressed before the clinical implications of this form of stunning can be fully appreciated and effective clinical therapies developed.

First, there is no direct evidence for a pathogenetic role of oxyradicals in myocardial stunning after recurrent ischemia. In this setting, neither the production of free radicals nor the ability of antioxidants to inhibit radical production has been documented. Furthermore, no previous study has assessed whether a sequence of recurrent episodes of ischemia and reperfusion results in recurrent bursts of free radical production and, if so, whether the intensity of these bursts increases, decreases, or remains unchanged as the number of repetitions increases. Moreover, the nature of the injurious radical species remains unknown. The beneficial effects of superoxide dismutase (SOD) ${ }^{1}$ plus catalase on stunning after repetitive ischemia (12) could be due to removal of superoxide radical $\left(\cdot \mathrm{O}_{2}^{-}\right)$and/or hydrogen peroxide $\left(\mathrm{H}_{2} \mathrm{O}_{2}\right)$ or, alternatively, to prevention of hydroxyl radical $(\cdot \mathrm{OH})$ formation (13). Also, if oxygen radicals contribute to this form of stunning, when does the injury responsible for contractile dysfunction occur? Does this injury occur mostly or entirely during the first reperfusion, so that the subsequent occlusion-reperfusion cycles add very little to the initial damage, or does it continue to occur with each successive reperfusion? This question also has practical implications because the duration of antioxidant therapy in patients with recurrent bouts of ischemia should depend on whether or not additional ischemic episodes are associated with additional oxidative stress.

The question of when the oxidative injury occurs is related to the more general issue of whether or not recurrent ischemic episodes have a cumulative effect on contractile function. This issue is important because, if the injury ceases after the first few ischemic episodes, then myocardial stunning would be a self-limiting process; on the other hand, if each subsequent ischemic episode continues to inflict additional injury, then the possibility exists that myocardium subjected to recurrent bouts of ischemia may become chronically stunned. Previous investigations have yielded conflicting results, with some $(6-10,14$ 22) but not all (23-25) studies reporting a cumulative effect. Even the studies that have observed a cumulative effect, however, have uniformly concluded that the decrement in function resulting from each subsequent occlusion becomes smaller with the number of repetitions and that after the first three to five occlusions, subsequent occlusions have little or no effect on postischemic dysfunction $(6-10,14-22)$. This has been interpreted as evidence for a preconditioning effect of the initial ischemic episodes.

One problem with the interpretation of these studies $(6-10$, 14-25) is that the presence or absence of a cumulative effect was judged solely from the nadir of function attained immediately or shortly after each ischemia-reperfusion cycle. To our knowledge, no previous study has systematically analyzed the effect of recurrent ischemic episodes on the duration and time course of postischemic abnormalities. Although recurrent isch-

1. Abbreviations used in this paper: EPR, electron paramagnetic resonance; LAD, left anterior descending coronary artery; MPG, mercaptopropionyl glycine; PBN, $\alpha$-phenyl $N$-tert-butyl nitrone; SOD, superoxide dismutase; $\mathrm{ThF}$, thickening fraction. emic insults may not exacerbate the mechanical dysfunction attained immediately or shortly after each reperfusion, they may increase the overall magnitude of postischemic dysfunction, i.e., the severity of the dysfunction present after the early period of reflow and/or the total duration of the dysfunction. Elucidation of this issue would require a different approach from that used in previous studies $(6-10,14-25)$; namely, different numbers of ischemic episodes would have to be produced in different groups of animals and integrated measurements of postischemic dysfunction would have to be compared.

The overall goal of this investigation was to perform a comprehensive analysis of the pathogenesis and pathophysiology of myocardial stunning after repetitive ischemia. The study had three specific objectives. The first was to test the hypothesis that oxygen radicals (in particular, $\cdot \mathrm{OH}$ ) contribute to the pathogenesis of myocardial stunning after recurrent ischemia. To this end, we initially evaluated the effects of mercaptopropionyl glycine (MPG), a potent and cell-permeant $\cdot \mathrm{OH}$ scavenger, on the recovery of myocardial function after 105 -min coronary occlusions followed by $4 \mathrm{~h}$ of reperfusion. To obtain direct evidence for the oxyradical hypothesis, we subsequently measured myocardial production of free radicals with the spin trap $\alpha$-phenyl $N$-tert-butyl nitrone (PBN) and electron paramagnetic resonance (EPR) spectroscopy in the presence and absence of MPG. Our second objective was to determine whether recurrent ischemic episodes have a cumulative effect on the postischemic depression of contractility. To this end, the overall magnitude of postischemic dysfunction (measured as the total postischemic deficit of wall thickening) was compared in three groups of dogs subjected to 1,3 , or 105 -min coronary occlusions. Having obtained evidence for a cumulative effect, our third objective was to elucidate whether the recurrent oxidative damage associated with each subsequent occlusion-reperfusion cycle was the mechanism responsible for such cumulative effect. To this end, the infusion of MPG was stopped after five or eight occlusionreperfusion cycles and the effect of the remaining five or two "unprotected" occlusions on postischemic dysfunction was evaluated.

\section{Methods}

A total of 117 dogs was used for this study. The experimental preparation and techniques have been described previously in detail (26-34).

\section{Phase A: studies of recovery of contractile function}

Experimental preparation. Pentobarbital-anesthetized dogs of either sex $(14-25 \mathrm{~kg}$ ) were instrumented as described previously (26-33). Fluid replacement, plasma $\left[\mathrm{K}^{+}\right]$, and basic physiologic variables were controlled as described (26-33).

Experimental protocol. Six groups of dogs (groups I-VI) were studied in phase A (Fig. 1). Groups I-IV underwent a sequence of 10 5-min left anterior descending coronary artery (LAD) occlusions, each separated by $10 \mathrm{~min}$ of reperfusion, followed by a final 4-h period of reperfusion (Fig. 1). Groups I-III received an infusion of MPG (Sigma Chemical Co., St. Louis, MO) at a dose of $50 \mathrm{mg} / \mathrm{kg} / \mathrm{h}$. MPG was dissolved in normal saline and infused through a peripheral vein at a rate of $25 \mathrm{ml} / \mathrm{h}$. MPG was selected because $(a)$ it is a powerful scavenger of - $\mathrm{OH}$ in vitro (29) and in vivo (33); (b) it has no effect on $\mathrm{O}_{2}^{-}$or $\mathrm{H}_{2} \mathrm{O}_{2}$ (29); and (c) it readily crosses cell membranes (35) and, therefore, may protect from oxyradicals generated both in the intracellular and in the extracellular space. In group I (also referred to as MPG-10 group), the infusion of MPG was started $15 \mathrm{~min}$ before the 1st coronary occlu- 


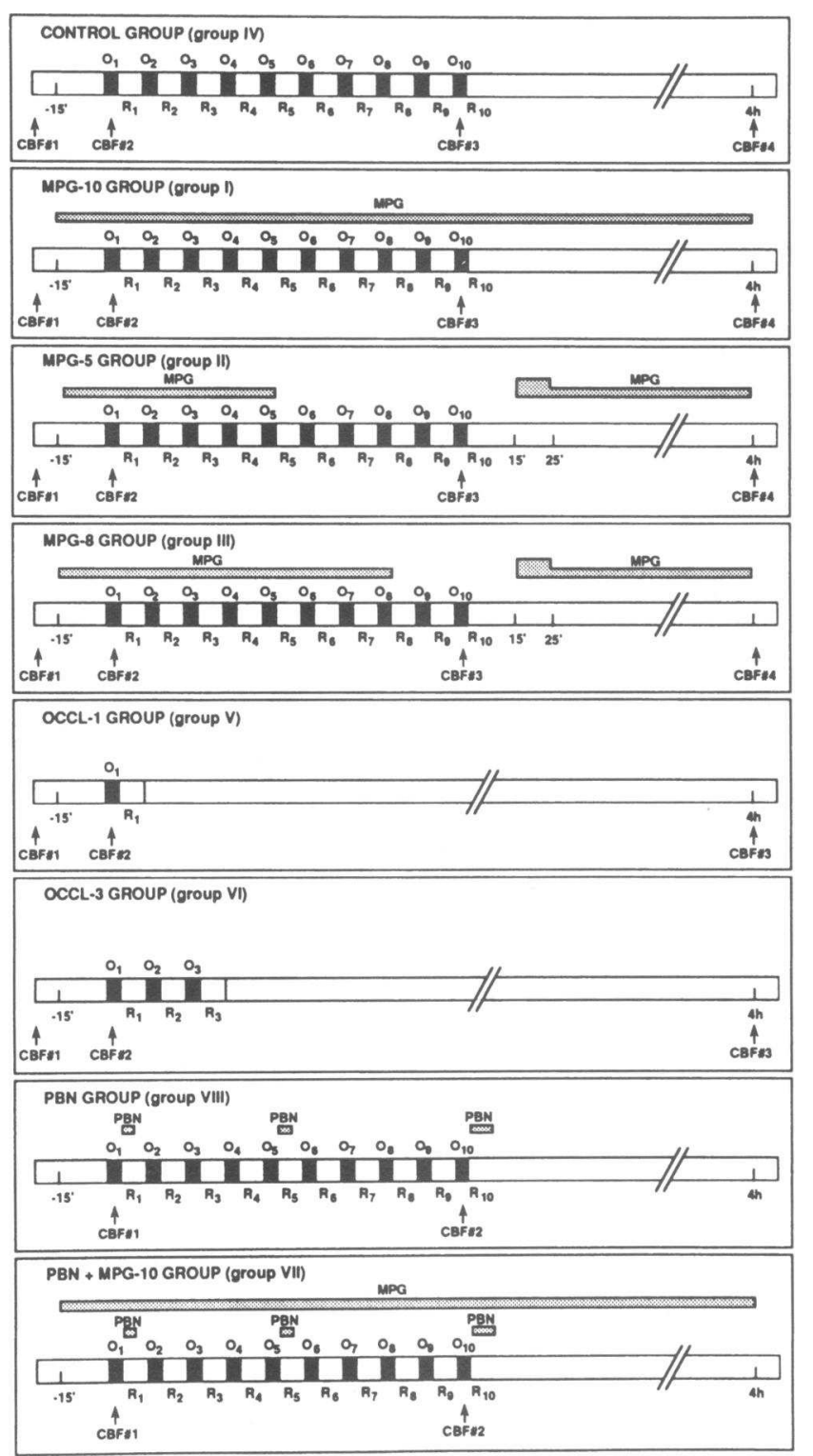

Figure 1. Schematic representation of the experimental protocols. Dogs were subjected to 1 (group V), 3 (group VI), or 10 (groups I-IV, VII, and VIII) 5-min LAD occlusions $(O)$ interspersed with $10 \mathrm{~min}$ of reperfusion $(R)$, followed by a final period of $4 \mathrm{~h}$ of reperfusion. Coronary blood flow $(C B F)$ was measured at the time points indicated by the arrows. Vehicle or MPG was given as a continuous intravenous infusion in groups I-IV, VII, and VIII, as indicated in the figure (in groups II and III, the rate of infusion of MPG was doubled between 15 and 25 min after the 10th reperfusion). In groups VII and VIII, PBN was infused intracoronarily starting $20 \mathrm{~s}$ before and ending $5 \mathrm{~min}$ after the 1st and 5th reperfusion and starting $20 \mathrm{~s}$ before and ending $10 \mathrm{~min}$ after the 10th reperfusion.

sion and continued until the end of the experiment $(4 \mathrm{~h}$ after the 10th reperfusion). In group II (MPG-5 group), the infusion of MPG was started $15 \mathrm{~min}$ before the first occlusion and stopped $1 \mathrm{~min}$ after the fifth reperfusion. The infusion was then restarted $15 \mathrm{~min}$ after the 10th reperfusion and continued until the end of the experiment. In group III (MPG-8 group), the infusion of MPG was started $15 \mathrm{~min}$ before the first occlusion and stopped $1 \mathrm{~min}$ after the eighth reperfusion. The infusion was then restarted $15 \mathrm{~min}$ after the 10th reperfusion and contin- ued until the end of the experiment. (To rapidly achieve plasma levels of MPG comparable to those in group I, in both groups II and III MPG was given at a rate of $100 \mathrm{mg} / \mathrm{kg} / \mathrm{h}$ during the first $10 \mathrm{~min}$ after restarting the infusion [i.e., between 15 and $25 \mathrm{~min}$ after the 10 th reperfusion], after which the rate was decreased to $50 \mathrm{mg} / \mathrm{kg} / \mathrm{h}$.) Thus, the difference among the three treatment groups was that in group I MPG was given continuously throughout the 10 occlusion-reperfusion cycles, whereas in groups II and III it was given only during the first 5 or 8 occlusionreperfusion cycles, respectively, so that the last 5 or 2 cycles were not "protected" by antioxidant therapy. Group IV (control group) received equivalent volumes of vehicle.

Group V (also referred to as OCCL-1 group) underwent one 5-min LAD occlusion followed by $4 \mathrm{~h}$ of reperfusion (Fig. 1). Group VI (OCCL-3 group) received a sequence of three 5-min LAD occlusions interspersed with $10 \mathrm{~min}$ of reperfusion, followed by a final 4-h interval of reperfusion (Fig. 1). These two groups were studied to compare the amount of stunning after 1,3 , and 10 brief coronary occlusions (groups $\mathrm{V}, \mathrm{VI}$, and IV, respectively).

In groups I-IV, regional myocardial blood flow was determined with radioactive microspheres (2) at baseline, during the 1st and 10th occlusion, and $4 \mathrm{~h}$ after the 10th reperfusion. In groups $\mathrm{V}$ and VI, microspheres were injected at baseline, during the first occlusion, and $4 \mathrm{~h}$ after the last reperfusion. Regional myocardial function was assessed as systolic thickening fraction (ThF) using a pulsed Doppler epicardial probe $(2,26-34)$. At the end of the study, the size of the occludedreperfused coronary vascular bed was determined by a postmortem dualperfusion technique (2).

Measurement of MPG plasma concentration. In dogs treated with MPG, venous blood samples $(5 \mathrm{ml})$ were obtained at selected times during the protocol. The plasma concentration of MPG was estimated from the total concentration of reduced nonprotein sulf hydryl groups (36), after subtracting the pre-MPG values, as described previously (34).

\section{Phase B: studies of free radical production}

Experimental preparation. The experimental preparation was similar to that used in phase A, with two differences. First, a 27-gauge needle connected to a lymphangiographic catheter was introduced into the LAD, just distal to the snare, for infusion of PBN (27). To prevent clotting, heparin (3,000 U intravenously) was given immediately after insertion of the needle and continuously thereafter $(500 \mathrm{U} / \mathrm{h})$. Second, a No. $8 \mathrm{~F}$ Sones catheter was introduced into the coronary sinus and advanced into the anterior interventricular vein, as described previously (27-30, 32-34).

Experimental protocol. Three groups of dogs (groups VII-IX) were studied in phase B. Groups VII and VIII underwent a sequence of 10 5 -min LAD occlusions, each separated by $10 \mathrm{~min}$ of reperfusion, as in phase A, and then were observed for $3 \mathrm{~h}$ after the last reperfusion (Fig. 1), whereas group IX served as a nonischemic control group. All three groups received PBN. PBN (Sigma Chemical Co.) was infused directly into the occluded coronary artery starting $20 \mathrm{~s}$ before and ending $5 \mathrm{~min}$ after the 1st reperfusion (infusion 1), starting $20 \mathrm{~s}$ before and ending $5 \mathrm{~min}$ after the 5 th reperfusion (infusion 2), and starting $20 \mathrm{~s}$ before and ending $10 \mathrm{~min}$ after the 10th reperfusion (infusion 3) (Fig. 1). In each dog, the rate of PBN administration (milligrams per minute) during the three subsequent infusions was identical. Before and after PBN administration, the artery was infused with an equivalent volume of vehicle. PBN was dissolved at a concentration of $5 \mathrm{mg} / \mathrm{ml}$ in a mixture of $80 \%$ normal saline and $20 \%$ water, so that the infusate was isosmotic with the plasma (osmolality $=284 \mathrm{mosmol} / \mathrm{kg}$ ) (27). Because LAD flow varied considerably from dog to dog, the rate of PBN infusion was normalized to flow, so as to achieve the same concentration of PBN $(285 \mathrm{mg} / \mathrm{ml}$ or $1.6 \mathrm{mM}$ ) in the coronary arterial blood in all dogs (27). Baseline coronary blood flow ranged from 11.9 to $40.0 \mathrm{ml} / \mathrm{min}$; the rate of PBN infusion varied accordingly from 3.6 to $12.0 \mathrm{mg} / \mathrm{min}$ (mean $=5.9 \pm 0.7[\mathrm{SEM}] \mathrm{mg} / \mathrm{min}$ ). The volume of solution infused (which 

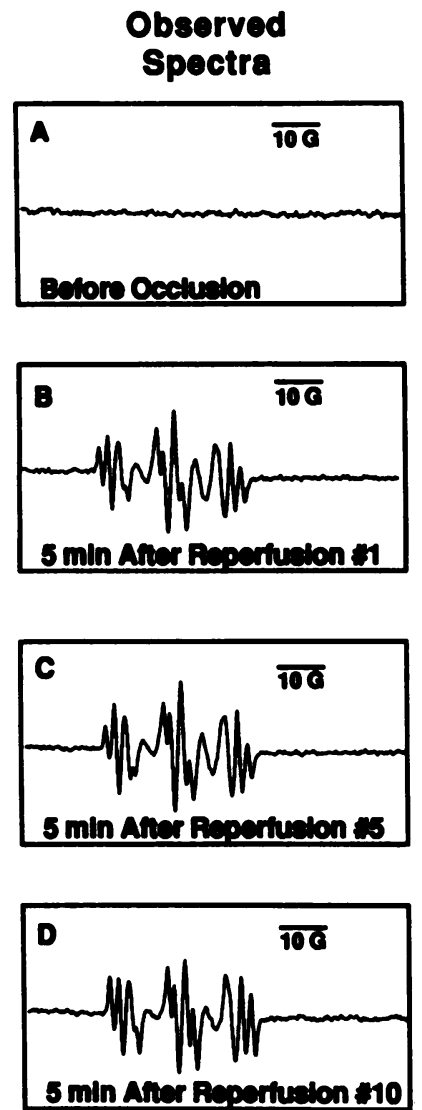

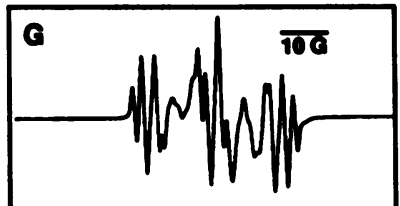

Simulated

Spectre
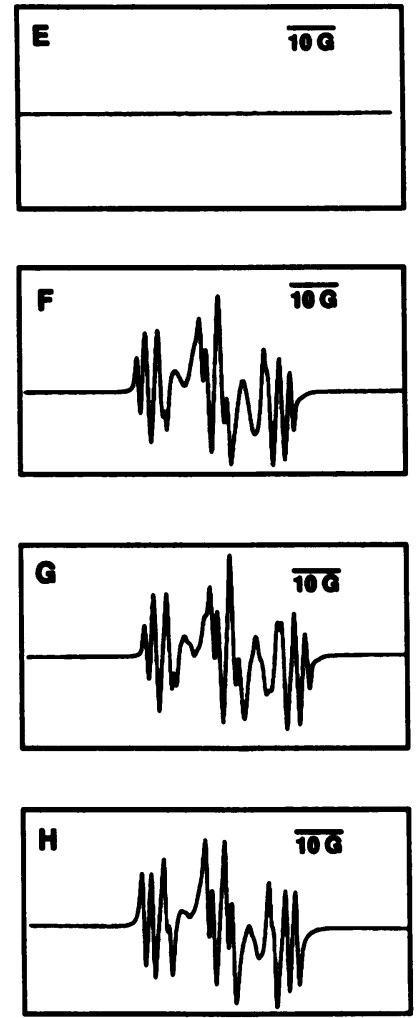

Observed Spectra
(MPG-Treated Dog)
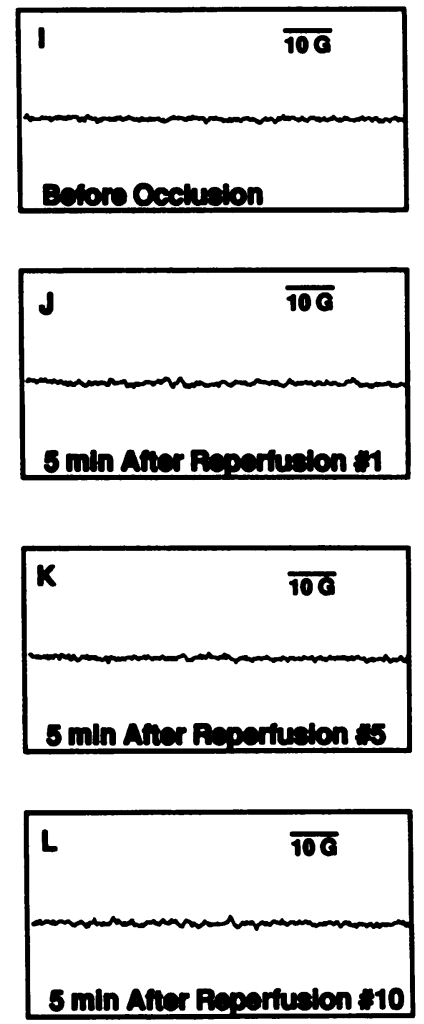

Figure 2. EPR spectra of spin adducts present in lipid extracts of plasma obtained from venous blood draining from the reperfused region. The horizontal bars above each spectrum indicate 10 G. The left column shows observed spectra in a dog in group IV (control group): $(A)$ before the 1st occlusion; $(B) 5$ min after the 1st reperfusion; $(C) 5 \mathrm{~min}$ after the 5 th reperfusion; and $(D) 5 \mathrm{~min}$ after the 10th reperfusion. The receiver gain was $2 \times 10^{6}$. The coupling constants for the various components were estimated by computer simulation as shown in Fig. 3. The center column, $E, F$, $G$, and $H$, shows computer-generated simulations of the observed spectra $A, B, C$, and $D$, respectively. The coupling constants for the various components in these simulated spectra are given in Fig. 3. The right column shows observed spectra in a dog in group I (treated with MPG throughout the 10 occlusion-reperfusion cycles): $(I)$ before the 1st occlusion; $(J) 5$ min after the 1st reperfusion; $(K)$ $5 \mathrm{~min}$ after the 5 th reperfusion; and $(L) 5 \mathrm{~min}$ after the 10th reperfusion. Receiver gain was $2 \times$ $10^{6}$. The spectrometer settings were as follows: microwave power, $20.0 \mathrm{~mW}$; modulation amplitude, $0.975 \mathrm{G}$; time constant, $1.31 \mathrm{~s}$; scan range, $100 \mathrm{G}$; and scan time, $356 \mathrm{~s}$. All spectra were recorded at room temperature $\left(25^{\circ} \mathrm{C}\right)$. ranged from 0.71 to $2.4 \mathrm{ml} / \mathrm{min}$ ) was equal to $6 \%$ of baseline coronary blood flow in all dogs. All animals were given PBN as described above and were assigned to three intravenous treatment groups. Group VII (also referred to as PBN + MPG-10 group) received MPG at the same dose previously used in phase A for group I, i.e., $50 \mathrm{mg} / \mathrm{kg} / \mathrm{h}$ starting $15 \mathrm{~min}$ before the first occlusion and continuing until the end of the experiment. Group VIII (PBN group) received equivalent volumes of the vehicle used for MPG. Group IX received PBN as in group VIII but did not undergo ischemia-reperfusion.

EPR analysis. Blood samples $(6 \mathrm{ml})$ were drawn over a 60 -s period from the aorta or from the anterior interventricular vein and immediately centrifuged. The plasma specimens were frozen at $-70^{\circ} \mathrm{C}$ for subsequent analysis by EPR spectroscopy. The techniques used to detect spin adducts of reactive free radicals and to quantify the myocardial release of these species into the coronary venous effluent blood have been described in detail $(27-30,32,34,37)$. The intensity of the EPR signals (which is proportional to the concentration of spin adducts in the sample) was expressed in arbitrary units; these were obtained by measuring (in millimeters) the cumulative height of the first three lines of the spectrum, adjusted for the gain of the scan (32) (Fig. 2). The first triplet was selected for these measurements because it was not affected by the (inconsistent) presence of $\alpha$-tocopheroxyl radicals (32) (Figs. 2 and 3; see Results). As indicated in Results, no adducts were detected in the arterial blood samples. Consequently, the myocardial release of spin adducts was calculated (in arbitrary units [U] per minute) by multiplying the signal intensity in the plasma of the venous effluent blood (which is proportional to the concentration of the adducts in that blood [arbitrary units per milliliter]) by the simultaneous LAD blood flow (milliliters per minute); this quantity was normalized to the size of the occluded-reperfused bed (measured in grams). Thus, the myocardial release of spin adducts at a specific time point was expressed in arbitrary units per minute per gram of reperfused myocardium (27-30, 32, 34, 37 ); the total cumulative myocardial release of PBN adducts over the first $5 \mathrm{~min}$ of reperfusion was calculated by integrating the measurements obtained at individual time points $(27-30,32,34,37)$.

Statistical analysis. All values are reported as means \pm SEM. Hemodynamic variables were analyzed by a two-way repeated-measures $\mathrm{AN}$ OVA (time and group) to determine whether there was a main effect of time, a main effect of group, or a time by group interaction. If the global tests showed a significant main effect or interaction, post-hoc contrasts between different time points within the same group or between different groups at the same time point were performed with Student's $t$ tests for paired or unpaired data, respectively, and the resulting $P$ values were adjusted according to the Bonferroni correction (38). The intensity of PBN adduct signals and the myocardial release of PBN adducts in groups VII and VIII were compared by nonparametric methods (Wilcoxon's signed rank test) because these data did not follow a normal distribution (38). The relationships between dependent and 

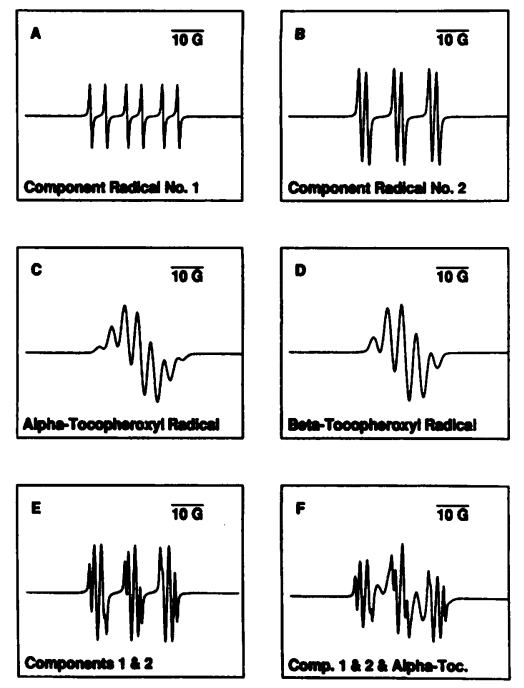

Figure 3. Computer-generated EPR spectra of component radicals believed to form the spectra shown in Fig. 2. The proportions of these various component radicals in each of the observed spectra in the left column of Fig. 2 are slightly different, resulting in some differences in peak intensities between samples. (A) Component 1, with coupling constants $a_{N}$ $=15.2 \mathrm{G}, \mathrm{a}_{\beta}{ }^{\mathrm{H}}=6.42 \mathrm{G}$; (B) component 2 , with coupling constants $\mathrm{a}_{\mathrm{N}}$ $=14.6 \mathrm{G}, \mathrm{a}_{\beta}{ }^{\mathrm{H}}=3.0 \mathrm{G}$; (C) component 3 , which is the $\alpha$-tocopheroxyl

radical, with coupling constants $\mathrm{a}^{\mathrm{CH} 3}(\mathrm{R} 1)=5.98 \mathrm{G}, \mathrm{a}^{\mathrm{CH} 3}(\mathrm{R} 2)=4.57$ $\mathrm{G}, \mathrm{a}^{\mathrm{CH} 3}(\mathrm{R} 3)=1.47 \mathrm{G}, \mathrm{a}^{\mathrm{CH} 2}=0.94 \mathrm{G} ;(D)$ component 4 , which is the $\beta$-tocopheroxyl radical with coupling constants $\mathrm{a}^{\mathrm{CH} 3}(\mathrm{R} 1)=6.2 \mathrm{G}$, $\mathrm{a}^{\mathrm{H}}(\mathrm{R} 2)=4.5 \mathrm{G}, \mathrm{a}^{\mathrm{CH} 3}(\mathrm{R} 3)=0.86 \mathrm{G}, \mathrm{a}^{\mathrm{CH} 2}=1.7 \mathrm{G} ;(E)$ combined spectra of components 1 and 2 at an area ratio of $1: 3$, respectively; $(F)$ combined spectra of components 1,2 , and $\alpha$-tocopheroxyl radical at an area ratio of $1: 3: 15$, respectively.

independent variables were analyzed using linear regression analysis with the least-squares method. All statistical analyses were performed with the SAS software system (39). Two-way ANOVA was performed using the procedure GLM (General Linear Models) (39).

\section{Results}

Phase A: studies of recovery of contractile function

\section{EXCLUSIONS}

Of the 98 dogs initially anesthetized in phase A, 23 (23\%) were excluded for the following reasons: death upon induction of anesthesia (one dog in group I and one in group III), technical problems (one dog in group I, one in group III, and one in group VI), lack of dyskinesis during ischemia (two dogs in group III), ventricular fibrillation during the first coronary occlusion (one dog in group II, two in group IV, and two in group V), ventricular fibrillation upon reperfusion (one dog in group I, one in group II, two in group III, three in group IV, one in group VI [ five of these dogs fibrillated after the first reperfusion, one after the second, one after the sixth, and one after the ninth]), spontaneous LAD occlusion after reperfusion (one dog in group I), and small ( $\leq 15 \%$ of left ventricular weight) occluded bed size (one dog in group I and one in group IV). Tetrazolium staining confirmed the absence of irreversible tissue damage in all animals, a finding consistent with previous studies in similar models $(6-10)$.

ARTERIAL BLOOD GASES, HEMATOCRIT, AND TEMPERATURE Arterial $\mathrm{pH}, \mathrm{PO}_{2}$, hematocrit, and esophageal temperature were within physiological limits in all groups throughout the experimental protocol (these data are not shown for the sake of brevity).
HEMODYNAMIC VARIABLES

With few exceptions, there were no significant differences in heart rate, arterial pressure, left atrial pressure, and left ventricular dP/dt among the six groups throughout the study (Table I). At baseline, the measurements of LAD blood flow (milliliters per minute) in groups I-VI averaged $16.3 \pm 1.6,18.2 \pm 2.5$, $19.7 \pm 2.2,19.8 \pm 3.5,22.9 \pm 2.8$, and $21.3 \pm 2.7$, respectively; at $1 \mathrm{~h}$ of reperfusion, $17.5 \pm 1.4,17.4 \pm 3.5,19.6 \pm 1.6,18.0 \pm 2.6$, $20.5 \pm 2.6$, and $20.3 \pm 2.3$, respectively; at $4 \mathrm{~h}$ of reperfusion, $18.1 \pm 1.8, \quad 17.3 \pm 2.7,21.8 \pm 2.2, \quad 17.2 \pm 4.1,22.1 \pm 2.7$, and $19.6 \pm 2.8$, respectively. There were no significant differences in LAD flow among the six groups at these three time points or at any other time point during the study. The rate-pressure product (heart rate $\times$ systolic pressure $/ 1,000$ ) also did not differ among the six groups (data not shown).

\section{OCCLUDED BED SIZE AND REGIONAL MYOCARDIAL BLOOD}

FLOW

The size of the occluded vascular bed was similar in all six groups (Table II). Compared with the 1st coronary occlusion, the average transmural ischemic zone flow during the 10th occlusion increased slightly in control dogs $(+16.7 \%)$ and in groups I and III ( +8.7 and $+38.5 \%$, respectively), whereas it decreased slightly in group II $(-12.1 \%)$ (Table III). Overall, these results indicate that the sequence of 10 occlusion-reperfusion cycles was not associated with significant changes in collateral perfusion. $4 \mathrm{~h}$ after reperfusion, the endocardial blood flow to the previously ischemic region was significantly less than the simultaneous flow to the nonischemic region in all groups that underwent 10 or 3 occlusions, whereas the epicardial flows were not lower (Table III). This pattern of selective depression of subendocardial perfusion in the stunned myocardium after multiple coronary occlusions is qualitatively and quantitatively similar to that observed previously after a 15 -min occlusion (2, $31,40)$. There were no significant differences among groups I-IV with respect to blood flow to the ischemic-reperfused region at any time point during the protocol, with the exception of endocardial flow $4 \mathrm{~h}$ after reperfusion, which was higher $(P$ $<0.05$ ) in groups I and III compared with controls (group IV) (Table III). Similarly, there were no significant differences among groups IV-VI with respect to blood flow to the ischemic-reperfused region at any time point in the course of the protocol (Table III).

\section{PLASMA CONCENTRATION OF MPG}

In group I, the concentration of MPG in the venous plasma rose progressively during the first five occlusion-reperfusion cycles and then remained relatively stable at $\sim 150 \mu \mathrm{M}$ throughout the rest of the experiment (Fig. 4). In group II, in which MPG was stopped $1 \mathrm{~min}$ after the 5 th reperfusion, the plasma levels of MPG were similar to those in group I until the 5th reperfusion but then decreased rapidly, so that at the time of the 9th and 10th reperfusions they averaged $\sim 10 \mu \mathrm{M}$. In group III, in which MPG was stopped $1 \mathrm{~min}$ after the 8th reperfusion, the plasma levels of MPG were similar to those in group I until the 8th reperfusion, but then fell to $53 \pm 12 \mu \mathrm{M}$ at the time of the 9th reflow and $29 \pm 7 \mu \mathrm{M}$ at the time of the 10th reflow. In both groups II and III, MPG infusion was restarted $15 \mathrm{~min}$ after the 10th reperfusion; this resulted in a prompt rise in MPG plasma concentration to values that were similar to those in group I throughout the rest of the reperfusion phase. Thus, the 


\begin{tabular}{|c|c|c|c|c|c|c|c|c|}
\hline & \multirow[b]{2}{*}{ Baseline } & \multirow[b]{2}{*}{$\mathrm{O}_{1}$} & \multirow[b]{2}{*}{$\mathbf{R}_{\mathbf{1}}$} & \multirow[b]{2}{*}{$\mathbf{R}_{\mathbf{1 0}}$} & \multicolumn{4}{|c|}{ Reperfusion } \\
\hline & & & & & $1 \mathrm{~h}$ & $2 \mathrm{~h}$ & $3 \mathrm{~h}$ & $4 \mathrm{~h}$ \\
\hline \multicolumn{9}{|l|}{ HR } \\
\hline MPG-10 (group I) & $156 \pm 5$ & $161 \pm 5$ & $163 \pm 5$ & $165 \pm 5$ & $170 \pm 5$ & $173 \pm 4$ & $174 \pm 5$ & $167 \pm 8$ \\
\hline MPG-5 (group II) & $170 \pm 7$ & $172 \pm 9$ & $169 \pm 9$ & $171 \pm 10$ & $184 \pm 5$ & $186 \pm 6$ & $188 \pm 5$ & $188 \pm 5$ \\
\hline MPG-8 (group III) & $159 \pm 5$ & $161 \pm 5$ & $161 \pm 5$ & $166 \pm 5$ & $170 \pm 5$ & $172 \pm 4$ & $174 \pm 5$ & $173 \pm 4$ \\
\hline Control (group IV) & $160 \pm 4$ & $155 \pm 5$ & $157 \pm 4$ & $161 \pm 5$ & $164 \pm 4$ & $165 \pm 4$ & $165 \pm 5$ & $164 \pm 7$ \\
\hline OCCL-1 (group V) & $158 \pm 5$ & $154 \pm 5$ & $155 \pm 5$ & - & $156 \pm 5$ & $154 \pm 6$ & $159 \pm 6$ & $160 \pm 6$ \\
\hline OCCL-3 (group VI) & $155 \pm 7$ & $153 \pm 6$ & $149 \pm 6$ & - & $154 \pm 6$ & $159 \pm 6$ & $165 \pm 6$ & $169 \pm 6$ \\
\hline \multicolumn{9}{|l|}{ SAP } \\
\hline MPG-10 (group I) & $134 \pm 4$ & $117 \pm 4$ & $119 \pm 3$ & $119 \pm 4$ & $118 \pm 5$ & $118 \pm 4$ & $115 \pm 4$ & $112 \pm 6$ \\
\hline MPG-5 (group II) & $141 \pm 8$ & $132 \pm 6$ & $134 \pm 7$ & $148 \pm 7 *$ & $138 \pm 5$ & $134 \pm 3$ & $128 \pm 4$ & $122 \pm 10$ \\
\hline MPG-8 (group III) & $124 \pm 5$ & $109 \pm 6^{*}$ & $112 \pm 5^{\ddagger}$ & $125 \pm 6$ & $121 \pm 7$ & $119 \pm 6$ & $116 \pm 8$ & $115 \pm 5$ \\
\hline Control (group IV) & $128 \pm 4$ & $124 \pm 4$ & $128 \pm 4$ & $127 \pm 4$ & $125 \pm 4$ & $123 \pm 4$ & $121 \pm 4$ & $119 \pm 5$ \\
\hline OCCL-1 (group V) & $121 \pm 6$ & $122 \pm 6$ & $124 \pm 6$ & - & $123 \pm 7$ & $124 \pm 6$ & $126 \pm 6$ & $122 \pm 6$ \\
\hline OCCL-3 (group VI) & $125 \pm 8$ & $121 \pm 9$ & $116 \pm 8$ & - & $114 \pm 8$ & $116 \pm 7$ & $119 \pm 8$ & $114 \pm 8$ \\
\hline \multicolumn{9}{|l|}{ LAP } \\
\hline MPG-10 (group I) & $5.0 \pm 0.7$ & $5.2 \pm 0.9$ & $4.4 \pm 0.9$ & $4.2 \pm 0.6$ & $4.0 \pm 0.7$ & $4.1 \pm 0.7$ & $4.5 \pm 1.1$ & $3.0 \pm 0.6^{8}$ \\
\hline MPG-5 (group II) & $5.2 \pm 0.5$ & $5.3 \pm 0.7$ & $4.8 \pm 0.7$ & $5.3 \pm 0.4$ & $5.2 \pm 0.5$ & $4.6 \pm 0.5$ & $4.2 \pm 0.6$ & $4.6 \pm 0.6$ \\
\hline MPG-8 (group III) & $4.8 \pm 0.3$ & $5.5 \pm 0.6$ & $5.2 \pm 0.4$ & $4.7 \pm 0.5$ & $4.4 \pm 0.6$ & $4.1 \pm 0.5$ & $4.0 \pm 0.5$ & $4.7 \pm 0.5$ \\
\hline Control (group IV) & $3.9 \pm 0.5$ & $6.6 \pm 1.0$ & $4.8 \pm 0.6$ & $3.5 \pm 0.5$ & $3.6 \pm 0.4$ & $3.6 \pm 0.4$ & $4.1 \pm 0.6$ & $4.8 \pm 0.6$ \\
\hline OCCL-1 (group V) & $4.6 \pm 0.5$ & $6.3 \pm 0.7$ & $5.4 \pm 0.6$ & - & $5.3 \pm 0.9$ & $5.2 \pm 0.7$ & $5.5 \pm 0.7$ & $5.2 \pm 0.7$ \\
\hline OCCL-3 (group VI) & $5.9 \pm 0.6^{\ddagger}$ & $6.6 \pm 0.8$ & $6.0 \pm 0.6$ & - & $6.0 \pm 0.6^{\ddagger}$ & $5.8 \pm 0.8^{*}$ & $5.6 \pm 0.8$ & $5.9 \pm 0.7$ \\
\hline \multicolumn{9}{|l|}{$\mathrm{LV} \mathrm{dP/dt_{ \operatorname {max } }}$} \\
\hline MPG-10 (group I) & $2682 \pm 251$ & $2549 \pm 255$ & $2547 \pm 298$ & $2752 \pm 299$ & $2944 \pm 304 *$ & $2882 \pm 341$ & $2840 \pm 289$ & $2867 \pm 238$ \\
\hline MPG-5 (group II) & $2164 \pm 407$ & $2219 \pm 404$ & $2274 \pm 440$ & $1999 \pm 432$ & $1972 \pm 373$ & $2192 \pm 337$ & $2109 \pm 277$ & $2329 \pm 348$ \\
\hline MPG-8 (group III) & $2233 \pm 194$ & $1990 \pm 174$ & $2020 \pm 164$ & $2257 \pm 157$ & $2364 \pm 156$ & $2407 \pm 189$ & $2439 \pm 192$ & $2579 \pm 172$ \\
\hline Control (group IV) & $2443 \pm 155$ & $2241 \pm 149$ & $2212 \pm 134$ & $2148 \pm 118$ & $2209 \pm 140$ & $2178 \pm 121$ & $2305 \pm 133$ & $2394 \pm 241$ \\
\hline OCCL-1 (group V) & $2050 \pm 169$ & $1967 \pm 246$ & $1883 \pm 128$ & - & $1967 \pm 111$ & $1911 \pm 116$ & $1950 \pm 144$ & $1939 \pm 169$ \\
\hline OCCL-3 (group VI) & $2359 \pm 238$ & $2145 \pm 220$ & $2086 \pm 188$ & - & $2155 \pm 175$ & $2209 \pm 187$ & $2282 \pm 259$ & $2164 \pm 250$ \\
\hline
\end{tabular}

Values are mean \pm SEM. $O_{1}$, 1st coronary occlusion; $R_{1}, 1$ st reperfusion; $R_{10}, 10$ th reperfusion; $H R$, heart rate (beats/minute); SAP, systolic arterial pressure $(\mathrm{mmHg}) ; L A P$, mean left atrial pressure $(\mathrm{mmHg}) ; L V d P / d t_{\max }$, maximal rate of left ventricular pressure rise $(\mathrm{mmHg} / \mathrm{second}){ }^{*} P<0.05$; ${ }^{8} P<0.02 ;{ }^{\ddagger} P<0.01$ vs. control.

protocols used for administration of MPG achieved the intended objective of decreasing the plasma levels of MPG to very low values during the last five or two occlusion-reperfusion cycles in groups II and III, respectively, while maintaining the MPG levels similar in all three groups during the rest of the protocol.

Table II. Size of the Occluded Vascular Bed

\begin{tabular}{llll}
\hline & LV (grams) & OB (grams) & OB (\% LV) \\
\hline MPG-10 (group I) & $81.0 \pm 4.2$ & $15.8 \pm 1.3$ & $19.7 \pm 1.4$ \\
MPG-5 (group II) & $83.3 \pm 5.3$ & $16.7 \pm 2.9$ & $20.4 \pm 3.7$ \\
MPG-8 (group III) & $78.9 \pm 3.3$ & $16.1 \pm 1.2$ & $20.6 \pm 1.5$ \\
Control (group IV) & $78.5 \pm 4.0$ & $15.3 \pm 1.2$ & $19.6 \pm 1.5$ \\
OCCL-1 (group V) & $89.4 \pm 4.1$ & $17.9 \pm 1.2$ & $20.0 \pm 1.1$ \\
OCCL-3 (group VI) & $76.0 \pm 4.6$ & $17.2 \pm 1.1$ & $23.1 \pm 1.4$ \\
PBN + MPG (group VII) & $89.5 \pm 15.6$ & $17.6 \pm 4.3$ & $19.5 \pm 3.2$ \\
PBN (group VIII) & $96.1 \pm 9.7$ & $21.9 \pm 2.8$ & $22.9 \pm 1.6$
\end{tabular}

Values are mean \pm SEM. $L V$, left ventricle; $O B$, occluded bed.
REGIONAL MYOCARDIAL FUNCTION

Studies of 105 -min occlusions ( groups I-IV). Baseline systolic $\mathrm{ThF}$ in the nonischemic (control) region averaged $17.3 \pm 1.4$, $14.5 \pm 4.1,14.9 \pm 1.6$, and $13.6 \pm 1.3 \%$ in groups I-IV, respectively; at $1 \mathrm{~h}$ of reperfusion, $\mathrm{ThF}$ in the nonischemic region was $84 \pm 7 \%$ of baseline values, $85 \pm 7,96 \pm 14$, and $106 \pm 13 \%$ in groups I-IV, respectively; at $4 \mathrm{~h}$ of reperfusion, it was $99 \pm 10 \%$ of baseline values, $90 \pm 9,92 \pm 12$, and $109 \pm 16 \%$, respectively. The values of $\mathrm{ThF}$ in the nonischemic (control) region did not differ significantly among the four groups at any time point during the protocol.

Baseline systolic ThF in the region to be rendered ischemic was $21.7 \pm 1.4 \%$ in group I, $26.6 \pm 2.4 \%$ in group II, $22.7 \pm 1.5 \%$ in group III, and $21.0 \pm 1.5 \%$ in group IV $(P=N S)$. For the sake of clarity, the 10 sequential measurements of ThF taken shortly ( $9 \mathrm{~min}$ ) after each of the 10 reperfusions are illustrated in Fig. 5, whereas the measurements taken during the occlusion phase and the following 4-h reperfusion interval are depicted in Fig. 6. In all four groups, the extent of paradoxical systolic thinning during ischemia did not change significantly with subsequent occlusions, so that during the 10th occlusion it was 
Table III. Regional Myocardial Blood Flow in Phase A of the Study

\begin{tabular}{|c|c|c|c|c|c|c|}
\hline & \multicolumn{3}{|c|}{ Ischemic zone } & \multicolumn{3}{|c|}{ Nonischemic zone } \\
\hline & Epi & Endo & Mean & Epi & Endo & Mean \\
\hline \multicolumn{7}{|c|}{ Baseline (ml/min per gram) } \\
\hline MPG-10 (group I) & $1.62 \pm 0.17$ & $1.47 \pm 0.19$ & $1.54 \pm 0.17$ & $1.26 \pm 0.14$ & $1.53 \pm 0.19$ & $1.40 \pm 0.16$ \\
\hline MPG-5 (group II) & $1.73 \pm 0.20$ & $1.54 \pm 0.11$ & $1.64 \pm 0.15$ & $1.44 \pm 0.18$ & $1.79 \pm 0.12$ & $1.61 \pm 0.15$ \\
\hline MPG-8 (group III) & $1.55 \pm 0.14$ & $1.44 \pm 0.16$ & $1.49 \pm 0.14$ & $1.38 \pm 0.12$ & $1.55 \pm 0.15$ & $1.46 \pm 0.13$ \\
\hline Control (group IV) & $1.69 \pm 0.18$ & $1.44 \pm 0.13$ & $1.57 \pm 0.15$ & $1.40 \pm 0.17$ & $1.60 \pm 0.17$ & $1.50 \pm 0.17$ \\
\hline OCCL-1 (group V) & $1.61 \pm 0.18$ & $1.44 \pm 0.15$ & $1.52 \pm 0.16$ & $1.28 \pm 0.11$ & $1.38 \pm 0.11$ & $1.33 \pm 0.11$ \\
\hline OCCL-3 (group VI) & $1.66 \pm 0.16$ & $1.56 \pm 0.13$ & $1.61 \pm 0.14$ & $1.48 \pm 0.13$ & $1.67 \pm 0.14$ & $1.58 \pm 0.13$ \\
\hline \multicolumn{7}{|c|}{ Occlusion $1(\mathrm{ml} / \mathrm{min} \text { per gram })^{*}$} \\
\hline MPG-10 (group I) & $0.31 \pm 0.06$ & $0.15 \pm 0.03$ & $0.23 \pm 0.04$ & $1.47 \pm 0.10$ & $1.55 \pm 0.11$ & $1.51 \pm 0.10$ \\
\hline MPG-5 (group II) & $0.47 \pm 0.12$ & $0.18 \pm 0.04$ & $0.33 \pm 0.07$ & $2.13 \pm 0.44$ & $2.48 \pm 0.49$ & $2.30 \pm 0.47$ \\
\hline MPG-8 (group III) & $0.36 \pm 0.11$ & $0.17 \pm 0.06$ & $0.26 \pm 0.08$ & $1.48 \pm 0.14$ & $1.52 \pm 0.16$ & $1.50 \pm 0.14$ \\
\hline Control (group IV) & $0.33 \pm 0.06$ & $0.14 \pm 0.03$ & $0.24 \pm 0.05$ & $1.76 \pm 0.24$ & $1.95 \pm 0.22$ & $1.86 \pm 0.23$ \\
\hline OCCL-1 (group V) & $0.32 \pm 0.07$ & $0.13 \pm 0.04$ & $0.22 \pm 0.05$ & $1.40 \pm 0.12$ & $1.43 \pm 0.12$ & $1.42 \pm 0.12$ \\
\hline OCCL-3 (group VI) & $0.42 \pm 0.11$ & $0.18 \pm 0.05$ & $0.30 \pm 0.08$ & $1.58 \pm 0.10$ & $1.75 \pm 0.12$ & $1.66 \pm 0.11$ \\
\hline \multicolumn{7}{|l|}{ Occlusion 1 (\% of NZF)* } \\
\hline MPG-10 (group I) & $23.0 \pm 5.8$ & $9.6 \pm 2.0$ & $16.0 \pm 3.6$ & - & - & - \\
\hline MPG-5 (group II) & $22.9 \pm 5.1$ & $8.5 \pm 2.2$ & $15.0 \pm 2.7$ & - & - & - \\
\hline MPG-8 (group III) & $23.6 \pm 7.6$ & $10.2 \pm 3.0$ & $16.3 \pm 5.0$ & - & - & - \\
\hline Control (group IV) & $26.7 \pm 6.4$ & $10.0 \pm 2.9$ & $17.7 \pm 4.4$ & - & - & - \\
\hline OCCL-1 (group V) & $24.2 \pm 4.6$ & $10.2 \pm 3.4$ & $17.1 \pm 3.9$ & - & - & - \\
\hline OCCL-3 (group VI) & $27.9 \pm 7.9$ & $10.8 \pm 3.9$ & $18.8 \pm 5.5$ & - & - & - \\
\hline \multicolumn{7}{|c|}{ Occlusion $10(\mathrm{ml} / \mathrm{min} \text { per gram })^{*}$} \\
\hline MPG-10 (group I) & $0.34 \pm 0.08$ & $0.15 \pm 0.03$ & $0.25 \pm 0.05$ & $1.34 \pm 0.07$ & $1.52 \pm 0.09$ & $1.41 \pm 0.08$ \\
\hline MPG-5 (group II) & $0.48 \pm 0.10$ & $0.10 \pm 0.02$ & $0.29 \pm 0.06$ & $1.58 \pm 0.41$ & $1.93 \pm 0.27$ & $1.76 \pm 0.34$ \\
\hline MPG-8 (group III) & $0.48 \pm 0.11$ & $0.24 \pm 0.07$ & $0.36 \pm 0.08$ & $1.64 \pm 0.19$ & $1.84 \pm 0.20$ & $1.74 \pm 0.20$ \\
\hline Control (group IV) & $0.39 \pm 0.07$ & $0.17 \pm 0.04$ & $0.28 \pm 0.06$ & $1.54 \pm 0.15$ & $1.93 \pm 0.19$ & $1.74 \pm 0.17$ \\
\hline OCCL-1 (group V) & - & - & - & - & - & - \\
\hline OCCL-3 (group VI) & - & - & - & - & - & - \\
\hline \multicolumn{7}{|c|}{ Occlusion 10 (\% of NZF)* } \\
\hline MPG-10 (group I) & $26.3 \pm 6.1$ & $9.7 \pm 1.8$ & $18.2 \pm 4.2$ & - & - & - \\
\hline MPG-5 (group II) & $32.4 \pm 7.5$ & $5.0 \pm 0.8$ & $17.1 \pm 3.3$ & - & - & - \\
\hline MPG-8 (group III) & $29.1 \pm 6.1$ & $12.7 \pm 3.4$ & $20.4 \pm 4.1$ & - & - & - \\
\hline Control (group IV) & $32.1 \pm 6.7$ & $10.5 \pm 2.6$ & $20.0 \pm 4.4$ & - & - & - \\
\hline OCCL-1 (group V) & - & - & - & - & - & - \\
\hline OCCL-3 (group VI) & - & - & - & - & - & - \\
\hline \multicolumn{7}{|c|}{ 4-h Reperfusion (ml/min per gram)* } \\
\hline MPG-10 (group I) & $1.71 \pm 0.21$ & $1.52 \pm 0.22^{\ddagger}$ & $1.62 \pm 0.21$ & $1.75 \pm 0.16$ & $1.80 \pm 0.14$ & $1.78 \pm 0.15$ \\
\hline MPG-5 (group II) & $1.85 \pm 0.21$ & $1.40 \pm 0.18^{\ddagger}$ & $1.62 \pm 0.19$ & $1.81 \pm 0.18$ & $1.94 \pm 0.25$ & $1.87 \pm 0.21$ \\
\hline MPG-8 (group III) & $1.77 \pm 0.19$ & $1.59 \pm 0.22^{\ddagger 8}$ & $1.68 \pm 0.20$ & $1.79 \pm 0.18$ & $1.84 \pm 0.22$ & $1.82 \pm 0.20$ \\
\hline Control (group IV) & $1.50 \pm 0.11$ & $1.10 \pm 0.07^{\ddagger}$ & $1.30 \pm 0.09^{\ddagger}$ & $1.61+0.15$ & $1.77+0.18$ & $1.69+0.17$ \\
\hline OCCL-1 (group V) & $1.68+0.20^{\ddagger}$ & $1.23 \pm 0.11$ & $1.46 \pm 0.15^{\ddagger}$ & $1.32 \pm 0.13$ & $1.28 \pm 0.13$ & $1.30 \pm 0.13$ \\
\hline OCCL-3 (group VI) & $1.60 \pm 0.15$ & $1.26 \pm 0.08^{\ddagger}$ & $1.43 \pm 0.11$ & $1.46 \pm 0.14$ & $1.44 \pm 0.12$ & $1.45 \pm 0.13$ \\
\hline \multicolumn{7}{|c|}{ 4-h Reperfusion (\% of NZF)* } \\
\hline MPG-10 (group I) & $97.6 \pm 6.1$ & $82.0 \pm 4.6^{8}$ & $89.4 \pm 4.7$ & - & - & - \\
\hline MPG-5 (group II) & $101.9 \pm 3.7$ & $72.6 \pm 3.8$ & $86.4 \pm 2.8$ & - & - & - \\
\hline MPG-8 (group III) & $100.2 \pm 6.7$ & $85.4 \pm 4.3^{8}$ & $92.5 \pm 4.8$ & - & - & - \\
\hline Control (group IV) & $99.7 \pm 5.9$ & $68.5 \pm 4.6$ & $83.2 \pm 4.9$ & - & - & - \\
\hline OCCL-1 (group V) & $125.9 \pm 4.4$ & $97.5 \pm 3.4$ & $111.7 \pm 3.4$ & - & - & - \\
\hline OCCL-3 (group VI) & $110.3 \pm 5.8$ & $89.5 \pm 4.3$ & $99.9 \pm 4.5$ & - & - & - \\
\hline
\end{tabular}

Values are mean \pm SEM. Endo, endocardial flow; Epi, epicardial flow; Mean, mean transmural flow; $N Z F$, simultaneous nonischemic zone flow. * Blood flow to the ischemic zone during coronary occlusion and $4 \mathrm{~h}$ after reperfusion is expressed both in absolute terms (milliliters per minute per gram) and as a percentage of NZF. ${ }^{\ddagger} P<0.05$ versus simultaneous nonischemic zone flow; ${ }^{8} P<0.05$ versus Control. 


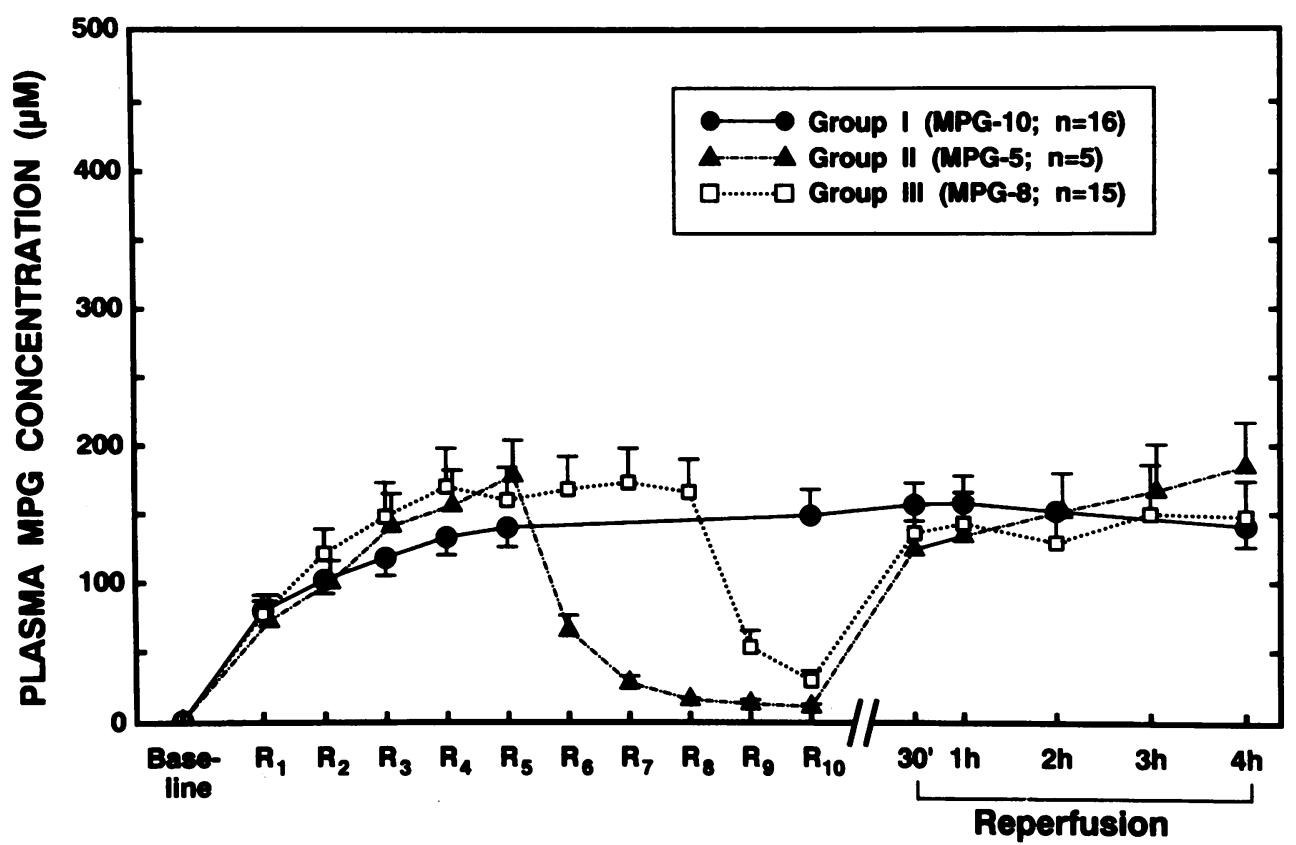

Figure 4. Graph showing the concentration of MPG in the venous plasma in groups I-III. Illustrated are measurements obtained at baseline (before infusion), $1 \mathrm{~min}$ into each of the 10 reperfusions after the 5-min LAD occlusions, and at selected times during the final 4-h reperfusion interval. Data are mean \pm SEM. similar to that measured during the 1st occlusion (Fig. 6). The analysis of postischemic regional function will be organized in two parts. First, we shall describe the sequential changes in $\mathrm{ThF}$ in control dogs (intragroup comparisons) and then we will compare this group with the three MPG-treated groups (intergroup comparisons).

(a) Changes within the control group. In control dogs (group IV), the first occlusion-reperfusion cycle caused the

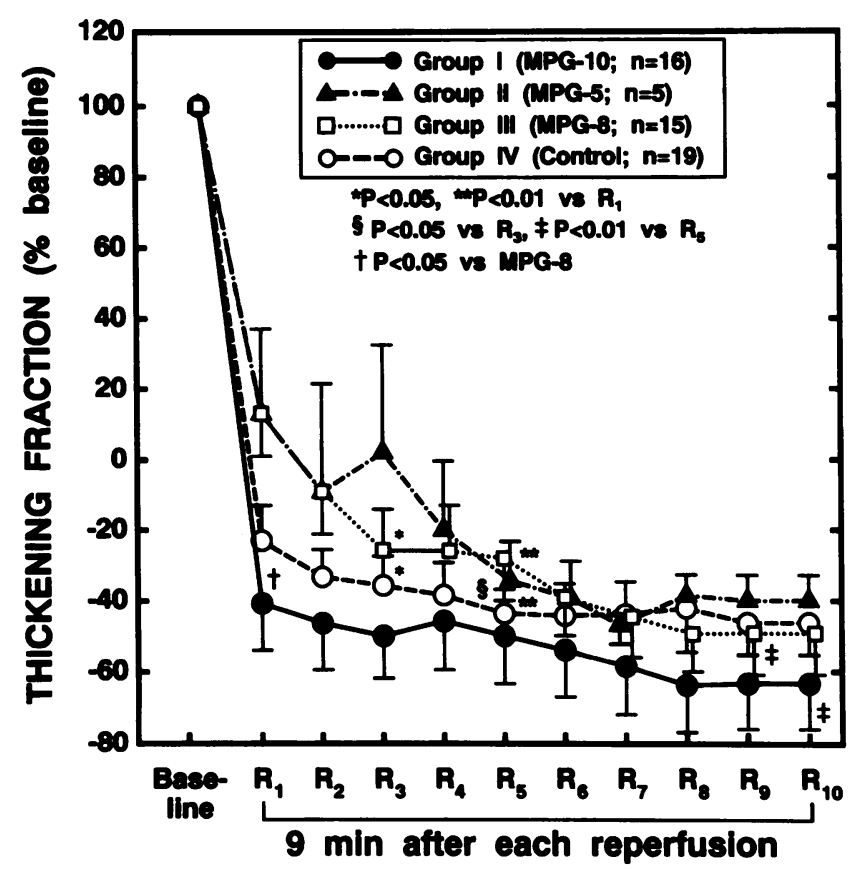

Figure 5. Graph showing the systolic $\mathrm{ThF}$ in the ischemic-reperfused region at baseline (before infusion of MPG or vehicle) and $9 \mathrm{~min}$ into each of the 10 reperfusion $(R)$ intervals after the 5-min occlusions. ThF is expressed as a percentage of baseline values. Data are mean \pm SEM. largest decrease in $\mathrm{ThF}$ (from baseline values to $-23 \%$ of baseline) (Fig. 5). During the subsequent four occlusion-reperfusion cycles, $\mathrm{ThF}$ exhibited a slight additional deterioration, reaching a nadir at $-44 \%$ of baseline during the fifth reflow (Fig. 5). The decrease in $\mathrm{ThF}$ between the first and third reperfusion was statistically significant $(P<0.05)$, and so was the decrease between the third and fifth reperfusion $(P<0.05)$. After the 5 th reperfusion, no further significant changes were noted, and

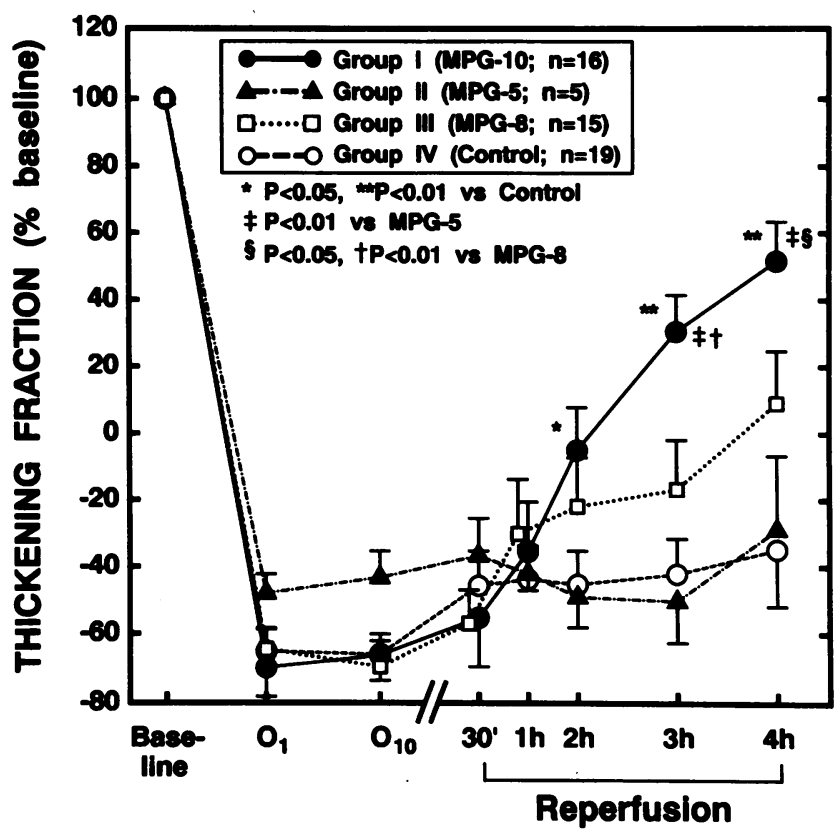

Figure 6. Graph showing the systolic $\mathrm{ThF}$ in the ischemic-reperfused region $4 \mathrm{~min}$ into the 1st $\mathrm{LAD}$ occlusion $\left(O_{1}\right), 4 \mathrm{~min}$ into the 10th LAD occlusion $\left(O_{10}\right)$, and at selected times during the final 4-h reperfusion interval. $\mathrm{ThF}$ is expressed as a percentage of baseline values. Data are mean \pm SEM. 


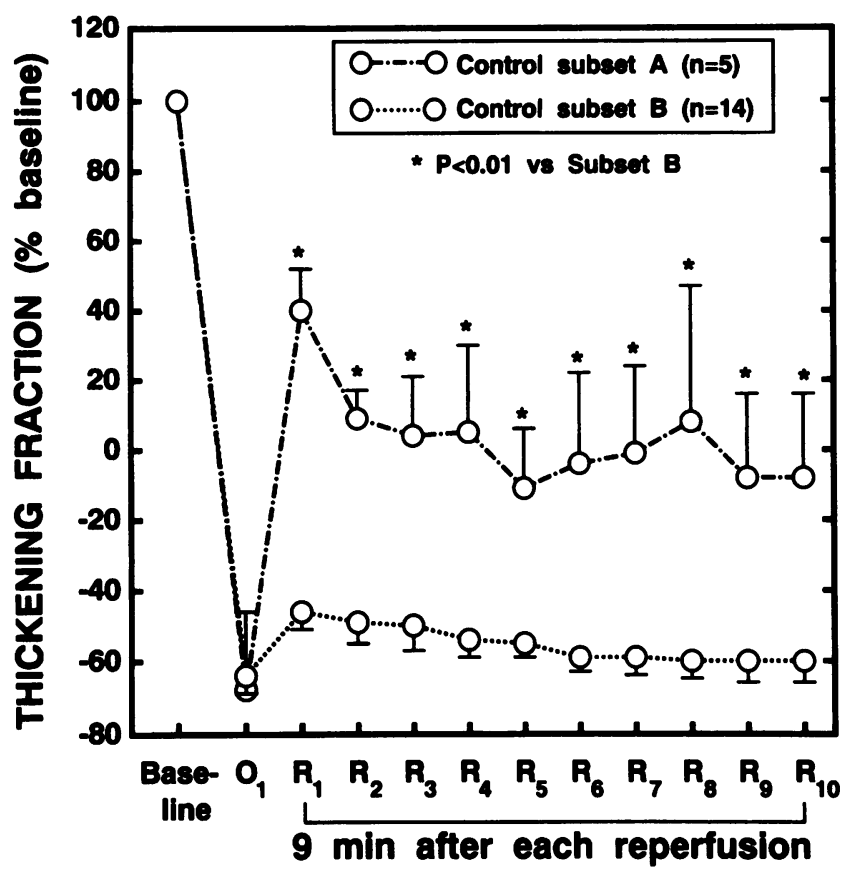

Figure 7. Graph showing the systolic ThF in the ischemic-reperfused region at baseline (before vehicle or MPG infusion), $4 \mathrm{~min}$ into the first LAD occlusion $\left(O_{1}\right)$, and 9 min into each of the 10 reperfusion $(R)$ intervals after the 5-min occlusions in two subsets of dogs within the control group (group IV): dogs in which the first reperfusion was associated with residual systolic wall thickening (hypokinesis) (subset $A, n=5$ ) and dogs in which the first reperfusion was associated with systolic wall thinning (dyskinesis) ( subset $B, n=14$ ). ThF is expressed as a percentage of baseline values. Data are mean \pm SEM.

ThF remained stable at $-45 \%$ of baseline values until the 10 th reperfusion (Fig. 5). Thus, judging from the measurements taken 9 min after reflow, the vast majority of the total postischemic loss of function developed during the first episode of ischemia and reperfusion, with only a slight additional loss developing during the next four episodes and no further loss during the last five episodes.

It may be argued, however, that the severity of dysfunction achieved after the first reperfusion was near maximal and prevented additional dysfunction from becoming manifest after the next nine reperfusions. To evaluate the validity of this argument, we repeated the same analysis in the subset of five control dogs (subset A) in which the first reperfusion was associated with residual wall thickening (hypokinesis) instead of wall thinning (dyskinesis) (Fig. 7). In these animals, ThF decreased to $40 \%$ of baseline values after the 1st reflow, $4 \%$ after the $3 \mathrm{rd}$, and $-11 \%$ after the 5 th, after which ThF remained stable at $\sim 0$ until the 10th reperfusion (Fig. 7). Even after the 10th reperfusion, the ThF measured in subset A was significantly higher than that $(\sim-60 \%$ of baseline) measured in the 14 control dogs in which the 1 st reperfusion was associated with dyskinesis (subset B) (Fig. 7). (Collateral flow was significantly higher in subset $A$ than in subset $B$ : $37 \pm 13$ vs. $11 \pm 2 \%$ of nonischemic zone flow, respectively, $[P<0.01]$ during the 1 st occlusion, and $41 \pm 12$ vs. $13 \pm 2 \%$ [ $P<0.01]$ during the 10 th occlusion.) Furthermore, in subset A the absolute decrease in systolic wall thickening associated with the 1st occlusion-reperfusion cycle was $1.00 \pm 0.26 \mathrm{~mm}$, whereas that associated with the 2 nd cycle was much smaller $(0.27 \pm 0.04 \mathrm{~mm})$, and those associated with the subsequent cycles (from the 3 rd to the 10th) were minimal $(<0.15 \mathrm{~mm})$. Thus, despite the fact that there was still "room" for further decreases in wall thickening in subset A, little or no additional dysfunction occurred after the initial two or three occlusion-reperfusion cycles. These findings are similar to those reported by Cohen and Downey (10). Taken together, the results obtained in subsets A and B and those obtained in the entire group indicate that, when function is measured shortly after reperfusion, the largest decrease occurs after the first occlusion-reperfusion cycle and the decreases associated with subsequent cycles become progressively smaller, to the point that after the fifth cycle recurrent ischemia produces no apparent change in function. These findings are ostensibly consistent with the concept that the first episode (or the first two to three episodes) of ischemia-reperfusion preconditions the myocardium against the stunning induced by further similar episodes.

After the sequence of 10 occlusion-reperfusion cycles, control dogs (group IV) exhibited little recovery of contractile function, and $4 \mathrm{~h}$ later the previously ischemic region was still dyskinetic ( $-35 \%$ of baseline) (Fig. 6$)$, indicating severe myocardial stunning.

After the first reperfusion, ThF in the stunned myocardium was directly related to collateral flow during the antecedent occlusion ( $r=0.64$, Fig. 8 ). The correlation between postischemic wall thickening and ischemic flow, however, became weaker after the second occlusion-reperfusion cycle $(r=0.46)$ and disappeared after the third $(r=0.27)$ (Fig. 8). Thereafter, no appreciable relationship was observed between wall thickening after reperfusion and blood flow during occlusion (Fig. 8).

(b) Comparisons among groups. During the first coronary occlusion, the extent of paradoxical systolic thinning was similar in groups I-IV (Fig. 6). Such similarity persisted during each of the subsequent nine occlusions (for the sake of clarity, only the 1st and 10th occlusions are depicted in Fig. 6). Likewise, 9 min after each of the 10 reperfusions, ThF did not differ significantly among the four groups (Fig. 5), with the exception of group $\mathrm{I}$, in which $\mathrm{ThF}$ was lower $(P<0.05)$ than in group III after the 1st reflow. Thus, during the sequence of 10 occlusionreperfusion cycles, administration of MPG had no immediate effect on the recovery of contractile function after each reperfusion.

A significant effect, however, was noted during the final 4$\mathrm{h}$ reperfusion interval (Fig. 6). In group I, which received MPG continuously throughout the protocol, the recovery of contractile function was substantially greater than in the control group at 2,3 , and $4 \mathrm{~h}$ after the 10th occlusion-reperfusion cycle (Fig. 6 ). In contrast, in group II, which received MPG during the first five but not during the last five occlusion-reperfusion cycles, the recovery of contractile function was minimal, and $\mathrm{ThF}$ was essentially indistinguishable from the control group (Fig. 6). In group III, which received MPG during the first eight but not during the last two occlusion-reperfusion cycles, the recovery of function tended to be greater than in the control group, but the differences did not achieve statistical significance at any time point (despite sample sizes of 15 and 19 dogs, respectively, in these two groups) (Fig. 6). Importantly, at 3 and $4 \mathrm{~h}$ of reperfusion, the measurements of $\mathrm{ThF}$ in group I were significantly greater than the corresponding values in both groups II 

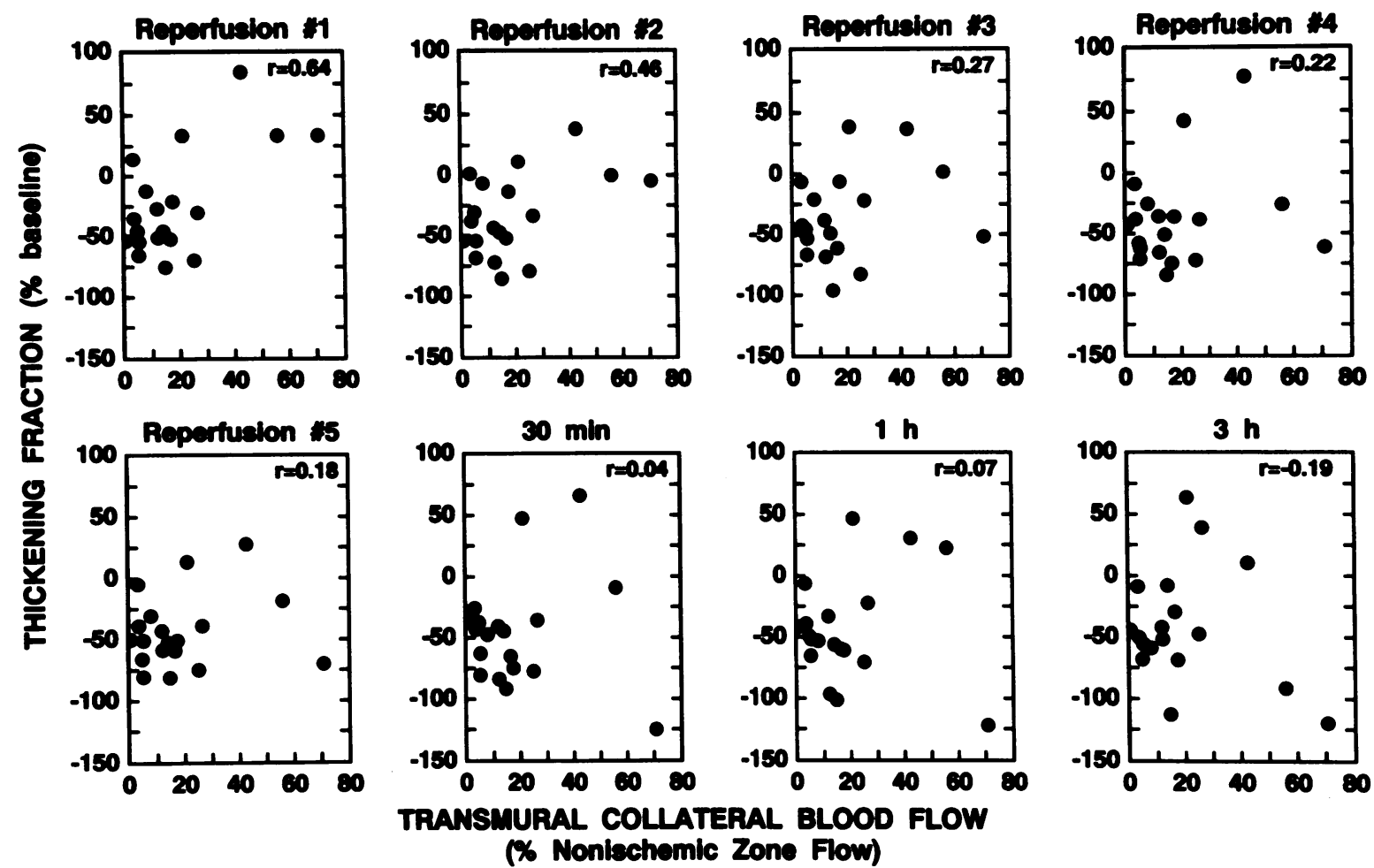

Figure 8. Plots showing the relation between mean transmural collateral blood flow to the ischemic region during coronary occlusion (horizonta axis) and systolic ThF (vertical axis) $9 \mathrm{~min}$ into the first, second, third, fourth, and fifth reperfusion intervals and $30 \mathrm{~min}, 1 \mathrm{~h}$, and $3 \mathrm{~h}$ into the final 4-h reperfusion interval in control dogs (group IV, $n=19$ ). Collateral flow is expressed as a percentage of simultaneous nonischemic zone flow, and ThF is expressed as a percentage of baseline values. The collateral flow depicted in all panels is that measured during the 1st LAD occlusion (the relationships are similar using the collateral flow measured during the 10th occlusion or the average of the collateral flows measured during the 1st and 10th occlusions). Note that there is a direct relationship between ThF and collateral flow during the first reperfusion, which becomes weaker after the second reperfusion, and disappears after the third. Thereafter, no appreciable relationship is observed between wall thickening after reperfusion and blood flow during occlusion.

and III (Fig. 6). These results indicate that, when myocardial stunning is induced by a sequence of 10 occlusion-reperfusion cycles, the beneficial effects of antioxidant therapy on the recovery of function can be completely (group II) or largely (group III) lost if the antioxidant is not administered during the last 5 or 2 cycles, respectively, implying that the oxidative stress associated with the last 5 , or even 2 , cycles of the sequence is sufficient to cause marked postischemic dysfunction. This finding is in contrast with the concept that the first few episodes of ischemia-reperfusion precondition the myocardium against the stunning induced by further similar episodes.

Comparisons of 1, 3, and 10 5-min occlusions (groups IVVI). Baseline systolic $\mathrm{ThF}$ in the nonischemic (control) region averaged $13.6 \pm 1.3,15.7 \pm 2.0$, and $19.1 \pm 1.8 \%$ in groups IVVI, respectively; at $1 \mathrm{~h}$ of reperfusion, $\mathrm{ThF}$ in the nonischemic region was $106 \pm 13 \%$ of baseline values, $93 \pm 7$, and $94 \pm 4 \%$ in groups IV-VI, respectively; at $4 \mathrm{~h}$ of reperfusion, it was $109 \pm 16,79 \pm 5$, and $81 \pm 5 \%$, respectively. The values of $T h F$ in the nonischemic (control) region did not differ significantly among the three groups at any time point during the protocol.

Baseline systolic ThF in the region to be rendered ischemic was $24.2 \pm 2.0 \%$ in group $\mathrm{V}, 24.0 \pm 1.2 \%$ in group VI, and $21.0 \pm 1.5 \%$ in group IV $(P=$ NS $)$. Fig. 9 illustrates the measurements of $\mathrm{ThF}$ taken during the first coronary occlusion and at serial times after the last reperfusion. All groups exhibited a similar degree of paradoxical systolic thinning during coronary occlusion (Fig. 9). The recovery of function during the final 4-h period of reperfusion did not differ significantly between group V, which underwent one 5-min occlusion, and group VI, which underwent three 5-min occlusions (Fig. 9). However, in group IV, which underwent 105 -min occlusions, the recovery of function was markedly less than that observed in either group V or VI (Fig. 9). The total deficit of wall thickening after reperfusion was calculated by measuring, with a computerized program, the area comprised between the thickening versus time line and the baseline ( $100 \%$ line) over the $4-h$ period of observation after the last reflow $(31,32,34)$ (Fig. 10). (This measurement integrates the deficit of function observed at individual time points and, therefore, provides an estimate of the overall magnitude of postischemic dysfunction during the entire reperfusion interval $[31,32,34]$.) As shown in Fig. 10, the total deficit of wall thickening over the final $4 \mathrm{~h}$ of reperfusion was not significantly greater in dogs subjected to 3 occlusions (group VI) compared with those subjected to 1 occlusion (group V); however, the deficit was considerably greater in dogs subjected to 10 occlusions (group IV), with a 2.5 -fold increase versus group VI $(P<0.01)$.

These results indicate that the second and third occlusionreperfusion cycles did not produce additional dysfunction compared with the first cycle (group VI versus V); thus, the first 


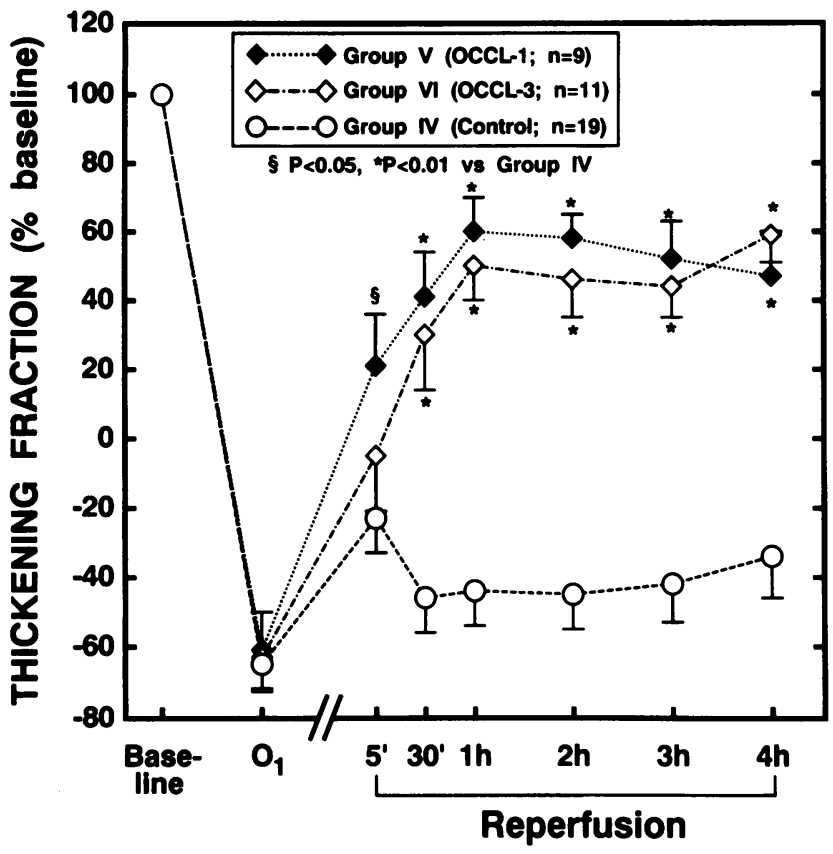

Figure 9. Graph showing the systolic ThF in the ischemic-reperfusion region at baseline, during the first LAD occlusion $\left(O_{I}\right)$ (in group $\mathrm{V}$, during the only LAD occlusion), and at selected times during the final 4-h reperfusion interval in group IV (control dogs subjected to 105 min occlusions), group V (dogs subjected to 15 -min occlusion), and group VI (dogs subjected to 35 -min occlusions). ThF is expressed as a percentage of baseline values. Data are mean \pm SEM.

cycle preconditioned the myocardium against the stunning induced by the next two cycles. This preconditioning effect, however, dissipated after the first 3 cycles, as demonstrated by the fact that when the total ischemic burden was tripled (from 3 to 10 occlusion-reperfusion cycles [groups VI and IV, respectively]) the overall magnitude of postischemic dysfunction almost tripled.

In group $\mathrm{V}$ there was a close direct correlation between $\mathrm{ThF}$ after reperfusion and collateral flow during occlusion $(r=0.87$ at $1 \mathrm{~h}$ and 0.89 at $2 \mathrm{~h}$, Fig. 11). To our knowledge, this is the first demonstration that contractile performance measured 1 or $2 \mathrm{~h}$ after a 5 -min coronary occlusion is directly related to ischemic flow. This correlation was much weaker after 3 occlusionreperfusion cycles (group VI; $r=0.44$ and 0.42 at 1 and 2 h, respectively, Fig. 11) and was completely absent after 10 occlusion-reperfusion cycles (group IV; $r=0.07$ and 0.10 at 1 and $2 \mathrm{~h}$, respectively; Fig. 8). These results are consistent with those obtained when wall thickening was measured $9 \mathrm{~min}$ after reperfusion (Fig. 8).

\section{Phase B: studies of free radical production}

For the sake of brevity, measurements in group IX are not reported.

\section{EXCLUSIONS}

Of the 19 dogs initially anesthetized in phase B, $5(26 \%)$ were excluded because of technical problems ( 2 dogs in group VII and 3 in group VIII). As in phase A, tetrazolium staining confirmed the absence of irreversible injury in all animals.
ARTERIAL BLOOD GASES, HEMATOCRIT, AND TEMPERATURE These variables were within physiological limits throughout the study in both groups VII and VIII (data not shown for the sake of brevity).

HEMODYNAMIC VARIABLES, OCCLUDED BED SIZE, AND MYOCARDIAL BLOOD FLOW

There were no significant hemodynamic differences between groups VII and VIII at any time point during the protocol (Table IV). (The rate-pressure product was also not significantly different [data not shown].) The two groups were also similar with respect to occluded bed size (Table II) and collateral blood flow (Table V).

\section{MYOCARDIAL PRODUCTION OF PBN ADDUCTS}

No PBN adducts were observed in preocclusion samples taken before the 1st, 5th, and 10th occlusions (Figs. 2 and 12). In group VIII (controls), EPR signals characteristic of radical adducts of PBN appeared in the coronary venous effluent immediately after the 1st, 5th, and 10th reperfusions (Figs. 2 and 12). The release of PBN adducts peaked at $1 \mathrm{~min}$ after the 1st and 5th reperfusion; after the 10th reperfusion, the release of PBN adducts peaked at 1 and 5 min and then declined, ceasing completely by $60 \mathrm{~min}$ (Fig. 12). No EPR signals were observed in any of the arterial blood samples (which were obtained at the same time as the venous samples), indicating that the presence of EPR signals in the venous blood cannot be ascribed to recirculation of PBN adducts. The total cumulative myocardial release of PBN adducts during the initial $5 \mathrm{~min}$ of reflow was $58 \%$ less after the 5 th reperfusion as compared with the 1st reperfusion $(753 \pm 125$ vs. $1,772 \pm 324 \mathrm{U} /$ gram, $P<0.05)$, but tended to be actually greater after the 10th reperfusion as compared with the 5th $(943 \pm 271$ vs. $753 \pm 125 \mathrm{U} /$ gram, $P=\mathrm{NS})$ (Fig. 13). The total release of PBN adducts in the initial 5 min of reflow did not differ significantly between the 10th and the 1st reperfusion $(P>0.20)$ (Fig. 13). These results indicate that recurrent brief ischemic episodes result in recurrent bursts of free radical production; although the magnitude of these bursts decreases between the 1st and 5th episodes it is not different between the 5th and 10th episodes, suggesting that the bursts continue to recur unabated during the last five occlusionreperfusion cycles.

In group VII, which received MPG throughout the experimental protocol, PBN adduct production was substantially decreased compared with control dogs (group VIII) (Figs. 2, 12, and 13). This effect was evident after the 1 st reperfusion and became even more pronounced after the 5 th and 10th reperfusions (Figs. 2, 12, and 13).

Group IX (control group for EPR signals) received three PBN infusions according to a protocol identical to that used in groups VII and VIII but did not undergo coronary occlusion. The three infusions of PBN did not produce any appreciable effect on regional contractile function or hemodynamic variables. No EPR signal was detected in any of the venous or arterial blood samples, which were obtained at times corresponding to those in groups VII and VIII.

\section{COMPUTER SIMULATION OF THE EPR SPECTRA}

Visual inspection of the EPR spectra observed (Fig. 2) indicates that the signals are likely due to a mixture of different radical adducts. In an effort to gain insights into the number, nature, and relative proportions of these adducts, computer simulation 

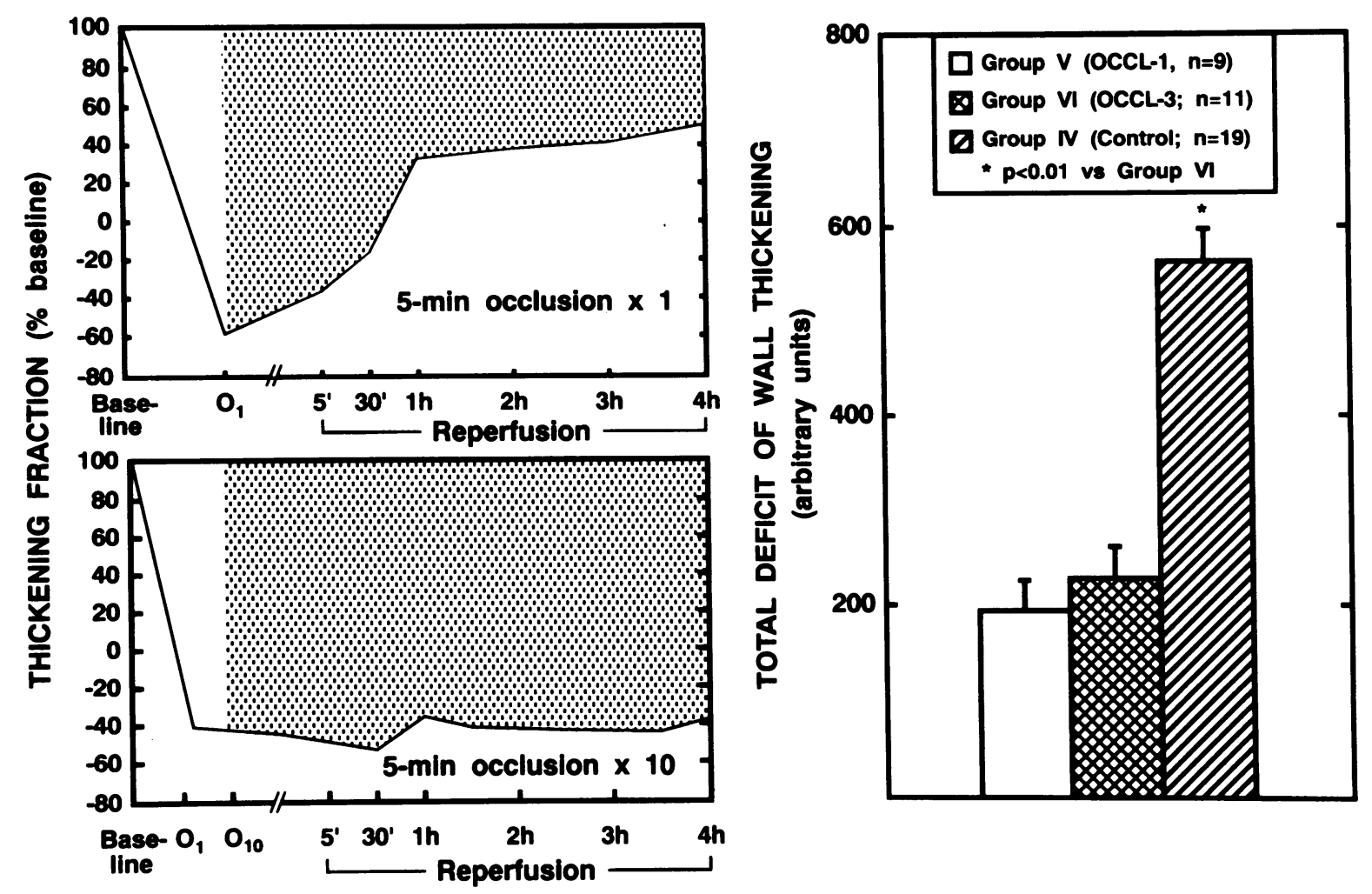

Figure 10. (Left) Graphs showing the systolic ThF in the ischemic-reperfused region at baseline, during the 1st LAD occlusion $\left(O_{l}\right)$, during the 10th LAD occlusion $\left(O_{10}\right)$, and at selected times during the final 4-h reperfusion interval in a dog in group V (1 5-min occlusion, top) and in a dog in group IV ( 105 -min occlusions, bottom). ThF is expressed as a percentage of baseline values. In both panels, the dotted area represents the area that is integrated to measure the total deficit of wall thickening after reperfusion. This area is comprised between the thickening versus time line and the baseline ( $100 \%$ line) over the 4-h period of observation after the last reperfusion. Measurement of this area provides an estimate of the overall magnitude of postischemic dysfunction during the entire reperfusion interval. (Right) The total deficit of wall thickening after reperfusion (measured as exemplified at the left) after 15 -min LAD occlusion (group V, open bar), 3 5-min occlusions (group VI, cross-hatched bar), and 105 -min occlusions (group IV, hatched bar). The total deficit of wall thickening is expressed in arbitrary units obtained by measuring the area of deficit with a computerized program. Values are mean \pm SEM.

of the spectra was performed as described previously $(28,29$, $32,37)$. The results of this analysis suggest the presence of at least four different components (Fig. 3): component 1, a PBN radical adduct with hyperfine coupling constants $\mathrm{a}_{\mathrm{N}}=15.2 \mathrm{G}$ and $\mathrm{a}_{\beta}{ }^{\mathrm{H}}=6.42 \mathrm{G}$; component 2 , a PBN radical adduct with $\mathrm{a}_{\mathrm{N}}$ $=14.6 \mathrm{G}$ and $\mathrm{a}_{\beta}{ }^{\mathrm{H}}=3.0 \mathrm{G}$; component 3 , which is the $\alpha$ tocopheroxyl radical; and component 4 , which is the $\beta$-tocopheroxyl radical. The release of tocopheroxyl radicals by the postischemic myocardium is consistent with the occurrence of oxidative stress upon reperfusion, since tocopherol is the major lipid-soluble endogenous antioxidant and is known to react with various radical species forming relatively persistent chromanoxyl radicals (41). For reasons that are unknown, the presence of tocopheroxyl radicals was erratic, with signals observed in some animals but not in others and, within the same animal, at some time points but not at others. Similar to the data previously obtained in conscious dogs (32), there was no correlation between the release of tocopheroxyl radicals and the release of PBN adducts (data not shown), suggesting that the former did not affect the latter. It should be noted that our method of measuring PBN adducts was not affected by the presence or absence of tocopheroxyl radicals (see Methods). The release of tocopheroxyl radicals was also unrelated to the collateral flow during ischemia or to the severity of postischemic dysfunction (data not shown) (32).

The combination of components $1,2,3$, and 4 resulted in computer-generated spectra (Fig. $2, F-H$ ) that closely resemble the corresponding observed spectra (Fig. 2, $B-D$ ). The four components were observed after each reperfusion but their relative proportions varied as the number of occlusion-reperfusion cycles increased, as illustrated in Fig. 2. For example, $5 \mathrm{~min}$ after the 1st reperfusion, components 1 and 2 were present in a ratio of $\sim 1: 3$ (Fig. 2, $B$ and $F$ ); 5 min after the 5th reperfusion, the ratio of component 1 to component 2 was still $\sim 1: 3$ (Fig. 2, $C$ and $G$ ), but $5 \mathrm{~min}$ after the 10th reperfusion it increased to $\sim$ 1:1 (Fig. $2, D$ and $H$ ). Components 1 and 2 continued to be present in ratios of $\sim 1: 1$ after the initial $5 \mathrm{~min}$ of the 10th reperfusion (data not shown). Thus, component 2 was the predominant PBN adduct released in the first part of the occlusion-reperfusion sequence (at least until the fifth reperfusion), whereas components 1 and 2 each accounted for approximately half of the adducts released at the end of the sequence.

The precise identity of components 1 and 2 cannot be ascertained from the present results. These two components are unlikely to be alkoxyl or peroxyl radicals because the PBN adducts 
$1 \mathrm{~h}$

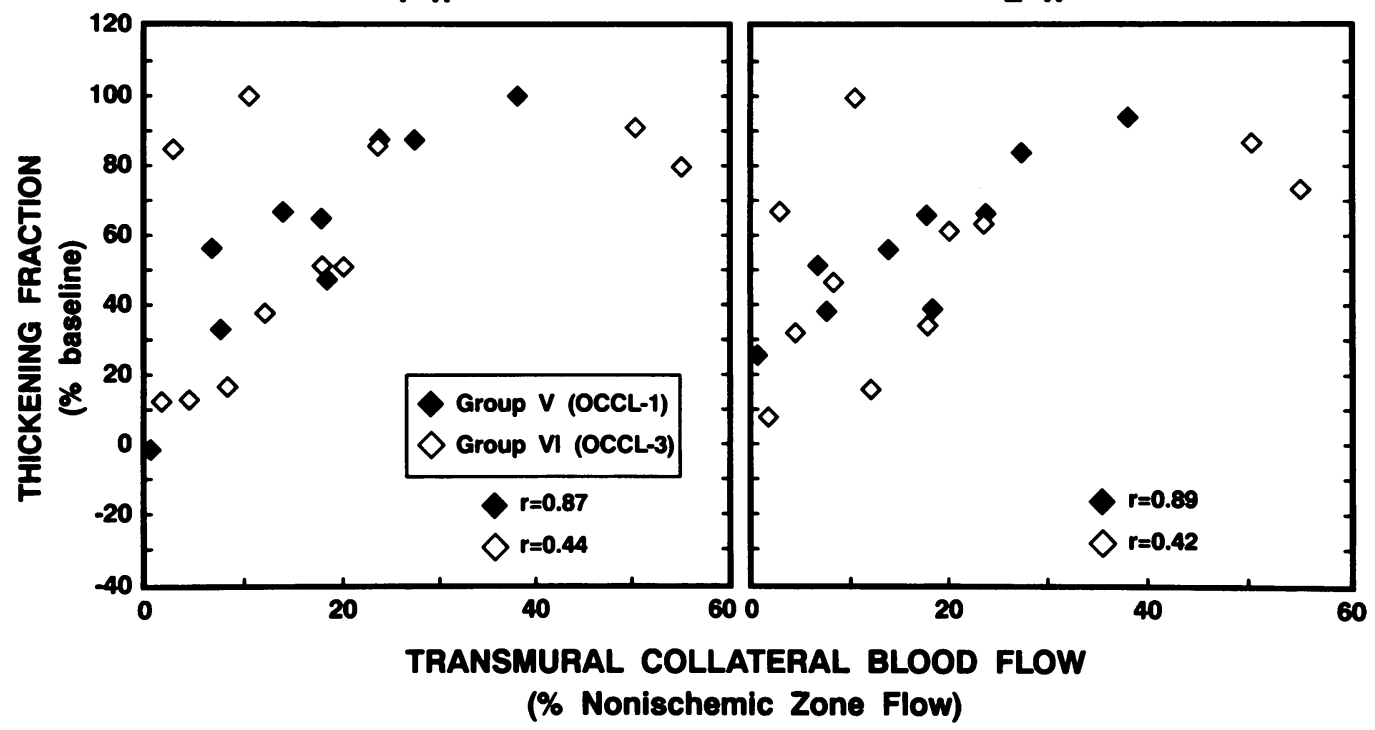

Figure 11. Plots showing the relation between mean transmural collateral blood flow to the ischemic region during the first LAD occlusion (horizontal axis) and systolic ThF (vertical axis) at 1 and $2 \mathrm{~h}$ after the last reperfusion in group V (one 5-min occlusion, solid diamonds) and group VI (three 5-min occlusions, open diamonds). Collateral flow is expressed as a percentage of simultaneous nonischemic zone flow, and ThF is expressed as a percentage of baseline values. Note that there is a close direct relationship between $\mathrm{ThF}$ after reperfusion and collateral flow during occlusion in group $\mathrm{V}$ and that this relationship is much weaker in group VI.

of these species usually have $a_{\beta}{ }^{H}<3.0 \mathrm{G}$ in chloroform (42). The hyperfine coupling constants of component $2\left(\mathrm{a}_{\mathrm{N}}=14.6\right.$ $\mathrm{G} ; \mathrm{a}_{\beta}{ }^{\mathrm{H}}=3.0 \mathrm{G}$ in chloroform) suggest the trapping of a carboncentered (alkyl) radical. The identity of component 1 (coupling constants: $\mathrm{a}_{\mathrm{N}}=15.2 \mathrm{G} ; \mathrm{a}_{\beta}{ }^{\mathrm{H}}=6.42 \mathrm{G}$ in chloroform) is more difficult to assign because of the unusually wide $\mathrm{a}_{\beta}{ }^{\mathrm{H}}$ (most of the known adducts of PBN have $\mathrm{a}_{\beta}{ }^{\mathrm{H}}<6.0 \mathrm{G}$ in chloroform).
It is possible that this component represents the adduct of a bulky molecule (such as a phospholipid, which in our experience can give PBN adducts with $\mathrm{a}_{\beta}{ }^{\mathrm{H}}>5.0 \mathrm{G}$ ). The fact that the PBN adducts seen in our study are soluble in nonaqueous solvents and resemble the lipid radical adducts of PBN observed in other systems (42) further supports the notion that they are derived from membrane lipids. These considerations, coupled

Table IV. Hemodynamic Variables in Phase B of the Study

\begin{tabular}{|c|c|c|c|c|c|c|c|c|}
\hline & \multirow[b]{2}{*}{ Baseline } & \multirow[b]{2}{*}{$\mathrm{O}_{1}$} & \multirow[b]{2}{*}{$R_{1}$} & \multirow[b]{2}{*}{$\mathrm{R}_{5}$} & \multirow[b]{2}{*}{$\mathbf{R}_{10}$} & \multicolumn{3}{|c|}{ Reperfusion } \\
\hline & & & & & & $1 \mathrm{~h}$ & $2 \mathrm{~h}$ & $3 \mathrm{~h}$ \\
\hline \multicolumn{9}{|l|}{ HR } \\
\hline PBN + MPG-10 (group VII) & $157 \pm 10$ & $168 \pm 6$ & $165 \pm 6$ & $161 \pm 5$ & $165 \pm 5$ & $164 \pm 9$ & $171 \pm 7$ & $166 \pm 9$ \\
\hline PBN (group VIII) & $157 \pm 8$ & $163 \pm 8$ & $161 \pm 7$ & $160 \pm 5$ & $162 \pm 8$ & $160 \pm 8$ & $160 \pm 8$ & $164 \pm 9$ \\
\hline \multicolumn{9}{|l|}{ SAP } \\
\hline PBN + MPG-10 (group VII) & $120 \pm 10$ & $102 \pm 7$ & $107 \pm 7$ & $94 \pm 9$ & $95 \pm 9$ & $84 \pm 11$ & $79 \pm 10$ & $80 \pm 9$ \\
\hline PBN (group VIII) & $115 \pm 5$ & $110 \pm 4$ & $106 \pm 6$ & $97 \pm 5$ & $91 \pm 6$ & $88 \pm 4$ & $89 \pm 3$ & $91 \pm 3$ \\
\hline \multicolumn{9}{|l|}{ LAP } \\
\hline PBN + MPG-10 (group VII) & $5.0 \pm 0.5$ & $4.8 \pm 0.7$ & $4.2 \pm 0.6$ & $3.4 \pm 0.2$ & $2.8 \pm 0.4$ & $2.5 \pm 0.3$ & $2.5 \pm 0.3$ & $3.5 \pm 1.0$ \\
\hline PBN (group VIII) & $3.7 \pm 0.4$ & $3.5 \pm 0.1$ & $3.8 \pm 0.3$ & $2.5 \pm 0.3$ & $2.2 \pm 0.7$ & $2.0 \pm 1.0$ & $1.8 \pm 0.6$ & $2.3 \pm 0.7$ \\
\hline \multicolumn{9}{|l|}{ LAD flow } \\
\hline PBN + MPG-10 (group VII) & $17.3 \pm 1.9$ & 0 & $19.3 \pm 2.8$ & $18.0 \pm 5.6$ & $16.3 \pm 4.4$ & $19.4 \pm 5.2$ & $18.6 \pm 3.6$ & $18.8 \pm 4.7$ \\
\hline PBN (group VIII) & $19.9 \pm 3.8$ & 0 & $21.7 \pm 7.4$ & $17.2 \pm 3.2$ & $21.7 \pm 7.3$ & $14.8 \pm 3.8$ & $15.6 \pm 4.3$ & $18.9 \pm 3.8$ \\
\hline \multicolumn{9}{|l|}{$\mathrm{LB} \mathrm{dP} / \mathrm{dt}_{\max }$} \\
\hline PBN + MPG-10 (group VII) & $1999 \pm 216$ & $1834 \pm 224$ & $1752 \pm 290$ & $1752 \pm 165$ & $1724 \pm 303$ & $1889 \pm 446$ & $1944 \pm 434$ & $1807 \pm 324$ \\
\hline PBN (group VIII) & $2146 \pm 250$ & $1894 \pm 226$ & $1905 \pm 163$ & $1527 \pm 152$ & $1527 \pm 148$ & $1504 \pm 170$ & $1676 \pm 196$ & $1779 \pm 170$ \\
\hline
\end{tabular}

Values are mean $\pm \mathrm{SEM} . O_{1}, 1$ st coronary occlusion; $R_{l}, 1$ st reperfusion; $R_{5}, 5$ th reperfusion; $R_{10}, 10$ th reperfusion; $H R$, heart rate (beats $\left./ \mathrm{min}\right) ; S A P$, systolic arterial pressure $(\mathrm{mmHg}) ; L A P$, mean left atrial pressure $(\mathrm{mmHg}) ; L A D$ flow, coronary blood flow in the left anterior descending artery $(\mathrm{ml} / \mathrm{min}) ; L V d P / d t_{\max }$, maximal rate of left ventricular pressure rise $(\mathrm{mmHg} / \mathrm{second})$. 
Table V. Regional Myocardial Blood Flow in Phase B of the Study

\begin{tabular}{|c|c|c|c|c|c|c|}
\hline & \multicolumn{3}{|c|}{ Ischemic zone } & \multicolumn{3}{|c|}{ Nonischemic zone } \\
\hline & Epi & Endo & Mean & Epi & Endo & Mean \\
\hline \multicolumn{7}{|l|}{ Occlusion $1^{*}$} \\
\hline \multicolumn{7}{|l|}{$\mathrm{ml} / \mathrm{min}$ per gram } \\
\hline PBN + MPG-10 (group VII) & $0.18 \pm 0.04$ & $0.07 \pm 0.02$ & $0.13 \pm 0.03$ & $1.40 \pm 0.29$ & $1.56 \pm 0.30$ & $1.49 \pm 0.30$ \\
\hline PBN (group VIII) & $0.14 \pm 0.03$ & $0.08 \pm 0.02$ & $0.11 \pm 0.03$ & $1.10 \pm 0.09$ & $1.24 \pm 0.07$ & $1.17 \pm 0.08$ \\
\hline \multicolumn{7}{|l|}{ Percentage of NZF } \\
\hline PBN + MPG-10 (group VII) & $15.1 \pm 5.2$ & $4.8 \pm 1.7$ & $9.5 \pm 3.0$ & - & - & - \\
\hline PBN (group VIII) & $12.7 \pm 3.0$ & $6.5 \pm 2.1$ & $9.4 \pm 2.3$ & - & - & - \\
\hline \multicolumn{7}{|l|}{ Occlusion $10^{*}$} \\
\hline \multicolumn{7}{|l|}{$\mathrm{ml} / \mathrm{min}$ per gram } \\
\hline PBN + MPG-10 (group VII) & $0.20 \pm 0.06$ & $0.09 \pm 0.03$ & $0.15 \pm 0.04$ & $1.53 \pm 0.43$ & $1.65 \pm 0.38$ & $1.59 \pm 0.40$ \\
\hline PBN (group VIII) & $0.16 \pm 0.04$ & $0.09 \pm 0.03$ & $0.12 \pm 0.04$ & $1.03 \pm 0.14$ & $1.11 \pm 0.14$ & $1.06 \pm 0.13$ \\
\hline \multicolumn{7}{|l|}{ Percentage of NZF } \\
\hline PBN + MPG-10 (group VII) & $19.4 \pm 8.1$ & $7.0 \pm 2.8$ & $12.5 \pm 4.9$ & - & - & - \\
\hline PBN (group VIII) & $14.7 \pm 2.9$ & $7.6 \pm 2.0$ & $11.1 \pm 2.5$ & - & - & - \\
\hline
\end{tabular}

Values are mean \pm SEM. Endo, endocardial flow; Epi, epicardial flow; Mean, mean transmural flow; $N Z F$, simultaneous nonischemic zone flow.

* Blood flow to the ischemic zone is expressed both as absolute terms (milliliters per minute per gram) and as percentage of NZF.

with the fact that the EPR spectra observed are not those of the $\cdot \mathrm{O}_{2}^{-}$or $\cdot \mathrm{OH}$ adducts, lead us to conclude that the radicals trapped by PBN in this study are secondary carbon-centered lipid radicals, such as alkyl radicals.

\section{Discussion}

The salient findings of this study can be summarized as follows: (a) the overall magnitude of postischemic dysfunction is similar after 1 and 35 -min coronary occlusions, but increases $\sim 2.5$ times after 10 occlusions; $(b)$ a coronary occlusion as short as $5 \mathrm{~min}$ is sufficient to cause a measurable burst of free radical production upon reperfusion; $(c)$ recurrent 5 -min occlusion/10min reperfusion cycles result in recurrent bursts of free radical generation; $(d)$ although the magnitude of these bursts decreases between the 1st and 5 th cycles, it is not decreased after the 10 th cycle as compared with the $1 \mathrm{st} ;(e)$ repetitive ischemia is associated with the generation of a mixture of carbon-centered (alkyl) spin-trapped radicals (most likely by-products of lipid peroxidation) and tocopheroxyl radicals; $(f)$ when given
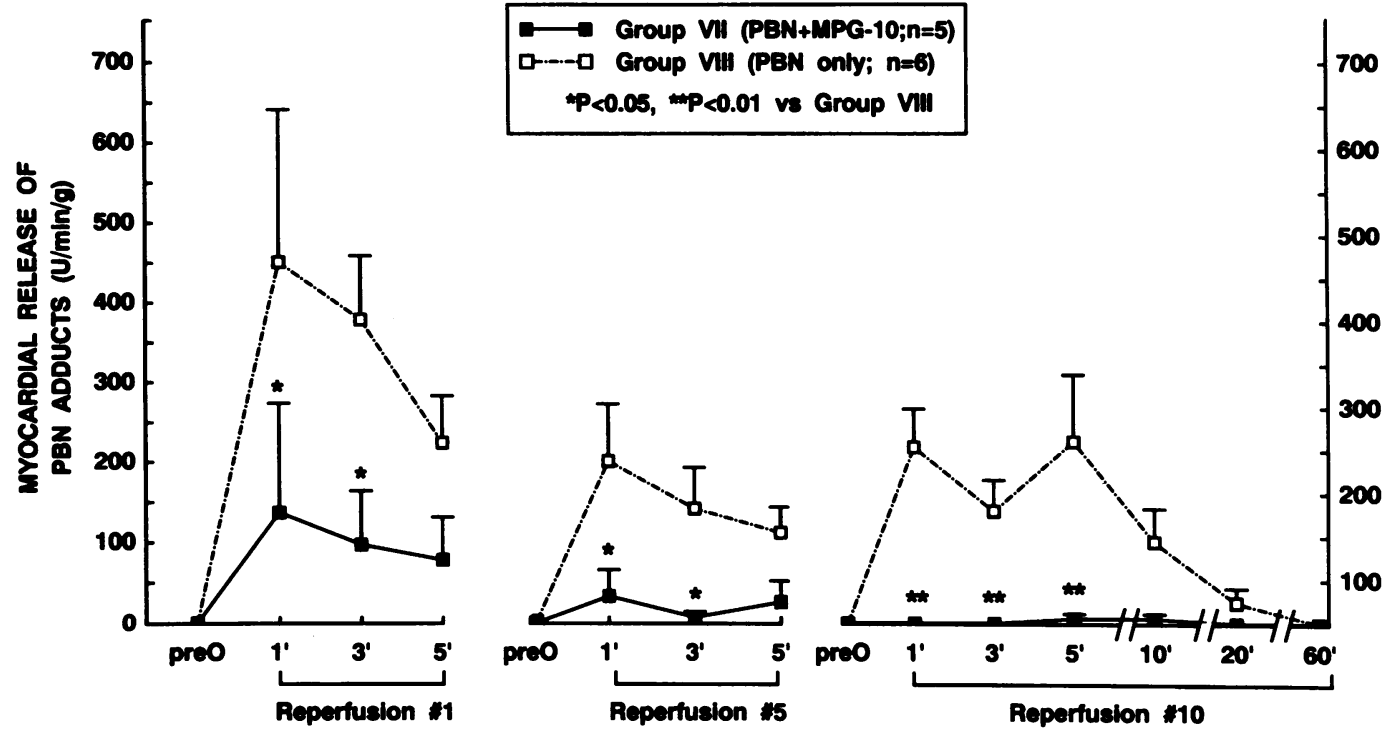

Figure 12. Graphs showing the myocardial release of PBN adducts in group VIII (control dogs) and group VII (MPG-treated dogs) in phase B of the study. Adduct release was measured at preocclusion ( $p r e O)$ (i.e., before the 1st, 5th, or 10th occlusion), and at 1,3 , and 5 min into the 1 st and 5th reperfusions, and 1, 3, 5, 10, 20, and $60 \mathrm{~min}$ into the 10 th reperfusion. Data are mean \pm SEM. See text for explanation of the units used. 


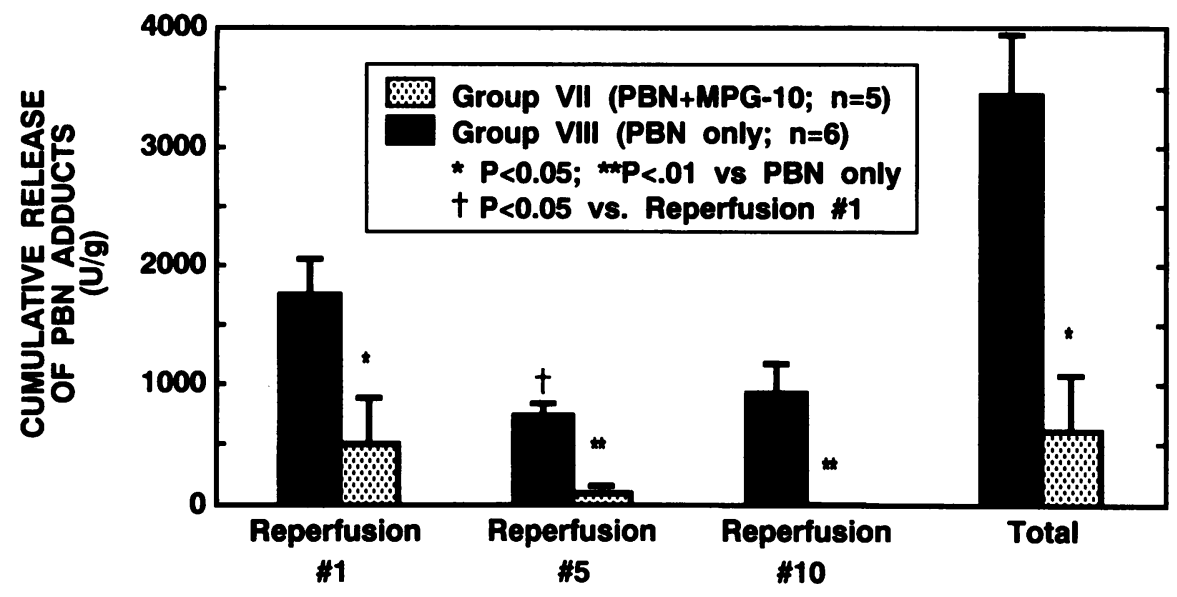

Figure 13. Bar graph showing the total cumulative myocardial release of PBN adducts during the first $5 \mathrm{~min}$ of reperfusion 1 , reperfusion 5, and reperfusion 10 in group VIII (control dogs, solid bars) and group VII (MPG-treated dogs, stippled bars). The bars on the right side illustrate the sum of the total cumulative myocardial release of PBN adducts during the first $5 \mathrm{~min}$ of the $1 \mathrm{st}, 5$ th, and 10th reperfusions. The cumulative release of PBN adducts is expressed in arbitrary units per gram of myocardium ( see text for explanation). Data are mean \pm SEM. throughout the 10 occlusion-reperfusion cycles, the $\cdot \mathrm{OH}$ scavenger MPG significantly enhances the recovery of function and inhibits the formation of free radicals; and $(g)$ the beneficial effects of MPG are completely, or largely, lost if the antioxidant is not given during the last 5 , or 2 , cycles, respectively. Taken together, these results indicate that the persistent depression of contractility (myocardial stunning) observed after recurrent brief ischemic episodes is due to the cumulative damage caused by the recurrent bursts of free radical generation associated with each episode of reperfusion.

First goal: pathogenesis of myocardial stunning after repetitive ischemia. The first goal of this study was to determine whether oxygen radicals contribute to the pathogenesis of myocardial stunning after recurrent ischemic episodes. Previous studies have suggested that oxyradicals play an important role in the genesis of myocardial stunning after a single, reversible ischemic insult (15-min coronary occlusion) (for review see reference 11). However, relatively little is known regarding the role of oxyradicals after multiple ischemic insults - a setting that is pathophysiologically different, as elaborated in the Introduction. Murry et al. (43) found that administration of SOD plus catalase failed to improve the recovery of function in openchest dogs subjected to four cycles of 5-min occlusion/5-min reperfusion; however, in this study dogs underwent a sustained 40-min occlusion shortly after the fourth reperfusion, so that there may not have been sufficient time to assess the presence of a protective effect. Similarly, Rohmann et al. (44) reported no effect of SOD plus catalase after two 10-min occlusion/30min reperfusion cycles in pigs, but the contractile recovery was followed for only $30 \mathrm{~min}$ after the second reperfusion. Using a longer (60 $\mathrm{min}$ ) reperfusion interval, Gross et al. (21) recently demonstrated that the contractile dysfunction caused by four cycles of 5-min occlusion/5-min reperfusion was significantly attenuated by the infusion of SOD plus catalase in open-chest dogs, indicating an important role of $\cdot \mathrm{O}_{2}^{-}$and/or $\mathrm{H}_{2} \mathrm{O}_{2}$. Nevertheless, direct evidence for a pathogenetic role of oxygen radicals in this model of myocardial stunning is still lacking. Furthermore, it is unknown whether $\cdot \mathrm{O}_{2}^{-}$and/or $\mathrm{H}_{2} \mathrm{O}_{2}$ causes dysfunction by direct cytotoxicity or indirectly, via formation of . OH (13).

We found that recurrent occlusion-reperfusion cycles were associated with recurrent bursts of free radical formation and that these bursts were markedly inhibited by the $\cdot \mathrm{OH}$ scavenger
MPG. Inhibition of radical generation by MPG resulted in attenuation of the ensuing postischemic dysfunction, indicating that free radical formation is necessary for the development of severe myocardial stunning in this setting. Taken together, our results support an important pathogenetic role of $\cdot \mathrm{OH}$ in this form of stunning. To our knowledge, this is the first demonstration that as little as $\mathbf{5} \mathrm{min}$ of regional ischemia is sufficient to induce free radical production in an intact animal. This study agrees with the results of Gross et al. (21) and expands our understanding of myocardial stunning in the following ways: $(a)$ It provides direct in vivo evidence that oxygen radicals mediate myocardial stunning after repetitive ischemia; $(b)$ it implicates the highly reactive $\cdot \mathrm{OH}$ as a primary culprit in this model of stunning; and $(c)$ it supports the concept that recurrent brief episodes of ischemia result in recurrent bouts of oxyradical-mediated injury that have a cumulative effect on contractility, a situation that could lead to protracted or even chronic stunning, as elaborated below.

Second goal: does repetitive ischemia have a preconditioning effect or a cumulative effect on myocardial stunning? The issue of whether recurrent ischemic episodes have a cumulative effect on postischemic dysfunction has been the focus of intense research because of its obvious clinical implications. The studies performed to date have yielded nonuniform results. Weiner et al. (23) measured regional myocardial function with segment-length gauges in open-chest dogs undergoing three consecutive 20-min coronary occlusions with $45 \mathrm{~min}$ of intermittent reperfusion. They found that ischemic segments recovered to the same level during each reperfusion period. Similarly, Lange et al. (24) measured segmental shortening and wall thickening in open-chest dogs subjected to three sequential 5- or 15min coronary occlusions separated by $30 \mathrm{~min}$ of reperfusion and concluded that the second and third ischemic episode caused no further dysfunction compared with the first episode. Using a canine model of stunning induced by six 5-min occlusion/ 10-min reperfusion cycles, Yao and Gross (25) found that the recovery of segment shortening after the sixth cycle was similar to that noted after the first cycle; in this study, a selective adenosine $\mathrm{A}_{1}$-receptor antagonist decreased the recovery of segment shortening after the first cycle but caused no additional deterioration after the next five cycles, suggesting that the adenosine released during the first ischemic episode was protective during that episode but did not precondition against the stunning 
induced by subsequent episodes. The absence of a cumulative effect appears also to be consistent with the finding that repetitive coronary occlusions are not associated with progressive depletion of high energy phosphates or significant cell death (45-48).

In contrast, Becker's group (6-9) reported that a sequence of 10-16 5-min coronary occlusions, each followed by $10 \mathrm{~min}$ of reperfusion, resulted in a progressive decrease in postischemic systolic shortening in open-chest dogs; the largest decrease occurred after the first occlusion, but subsequent ischemic periods led to additional, albeit progressively smaller, decrements. Similar results were obtained by Hoffmeister et al. (14) in openchest dogs subjected to 40 cycles of 3-min coronary occlusion/ 3-min reperfusion. A cumulative deterioration of contractile function has also been observed in open-chest dogs after three cycles of 5-min occlusion/45-min reperfusion or 15-min occlusion/45-min reperfusion (16), after four cycles of 5-min occlusion/45-min reperfusion or 15-min occlusion/45-min reperfusion (16), after four cycles of 5-min occlusion/5-min reperfusion (21), and after four cycles of 12-min occlusion/30 min reperfusion (20); in open-chest pigs after four cycles of 15min occlusion/45-min reperfusion (15) and after two cycles of 10-min occlusion/30-min reperfusion (18); and in conscious dogs after three episodes of exercise-induced ischemia (17). In one study (19) in conscious dogs subjected to five 5-min LAD occlusions, a cumulative effect was found when the intervening reperfusion period was $\leq 10 \mathrm{~min}$ but not when it was $15 \mathrm{~min}$. Recently, Cohen and Downey (10) performed a detailed analysis of the changes in regional function after 125 -min occlusions alternated with $10 \mathrm{~min}$ of reflow in open-chest dogs. They noted that with successive occlusion-reperfusion cycles the recovery of systolic shortening decreased progressively; however, the rate of deterioration was markedly attenuated after the first cycle, and in hearts in which active contraction was preserved there was no significant change in regional function after the third cycle, suggesting a protective or preconditioning effect of early ischemia (10). This conclusion is further bolstered by the findings of Bunch et al. (22). Using a rabbit model of stunning induced by four cycles of 5-min coronary occlusion/10-min reperfusion, these authors found that an adenosine receptor antagonist caused a progressive deterioration of function after each cycle, suggesting that the adenosine produced during the first ischemic episode preconditioned the myocardium against the subsequent episodes.

The reason for the discrepancy between the studies by Weiner et al. (23), Lange et al. (24), and Yao and Gross (25), on the one hand, and the other investigations $(6-10,14-18$, 20-22), on the other hand, is not clear. In any case, the bulk of the available evidence $(6-10,14-22)$ indicates that repetitive ischemic episodes have a cumulative effect on contractility. The same evidence (6-10,14-22), however, also indicates that the decrement in function becomes progressively smaller with each recurrent ischemic episode. This fact has been generally interpreted as evidence that the amount of injury caused by each occlusion-reperfusion cycle decreases with subsequent cycles, perhaps because the initial ischemic episodes render the myocardium more resistant to ischemia (10), a phenomenon that would be analogous to the preconditioning effect against cell death (49). If this hypothesis were correct, myocardial stunning would be a self-limiting process: myocardium stunned by an initial ischemic episode would be protected (preconditioned) against subsequent stunning so that any cumulative effect would cease after the first few (three to five) ischemic episodes.

The second goal of our study was to evaluate this hypothesis. In accordance with previous studies $(6-10,14-22)$, we found that the first occlusion caused the largest decrease in function (Fig. 5), that subsequent occlusions caused only relatively small additional decrements (Fig. 5), and that this pattern could not be explained by the fact that postischemic dysfunction had reached near-maximal levels after the first reperfusion (Fig. 7). On the basis of these data alone, we would have to conclude, as did the previous reports, that the last five occlusion-reperfusion cycles did not produce any additional dysfunction, i.e., that the myocardium was preconditioned up until the 10th occlusion. In the present study, however, we used a different approach to determine whether there was a cumulative effect. Instead of evaluating the recovery of function shortly after each reperfusion (as has been done thus far $[6-10,14-25]$ ), we evaluated the recovery of function over the entire 4-h reperfusion period after the last occlusion. We reasoned that a single measurement taken during a relatively unstable phase (early reflow) may not necessarily reflect the subsequent time course of myocardial stunning and that a more accurate approach would be to measure the severity and duration of stunning for several hours after reperfusion.

The results of this analysis differ from those suggested by Fig. 5 and by previous studies $(6-10,14-25)$. We found that the overall magnitude of postischemic dysfunction (as assessed by the total deficit of wall thickening) was similar after one and three 5-min occlusions (Figs. 9 and 10), indicating that the first ischemic episode did precondition against the next two episodes, in agreement with previous interpretations $(10,22$, $25)$. However, the overall magnitude of postischemic dysfunction was much greater after 10 occlusions than after 3 (Figs. 9 and 10), indicating that the last 7 occlusions caused considerable additional injury; that is, the first 3 ischemic episodes failed to precondition against the next 7 episodes. We therefore conclude that in the setting of recurrent brief coronary occlusions the protection against stunning afforded by ischemic preconditioning exists but is limited; at some point between the 4th and 10 th occlusions, this protection is lost, and recurrent ischemic episodes start to have a cumulative effect.

These conclusions are in contrast with those that we would have made on the basis of the data depicted in Fig. 5. Accordingly, we propose that the magnitude of the injury caused by recurrent ischemic insults cannot be adequately assessed from the magnitude of the dysfunction attained shortly after each insult. We suggest that the controversy regarding the cumulative effect of repetitive ischemia stems in part from the fact that the presence or absence of such an effect has been inferred primarily from the degree of dysfunction measured after a few minutes of reperfusion rather than from the overall severity of postischemic dysfunction.

The loss of protection against stunning after the first 3 occlusions may be due to the fact that the preconditioning effect is limited either in its efficacy (i.e., it protects only against a limited ischemic burden, less than that of 105 -min occlusions) or in its duration (i.e., it lasts less than the time [150 $\mathrm{min}$ ] necessary to complete the 10-occlusion sequence). In either case, preconditioning against stunning resembles preconditioning against cell death (50). The present study does not provide data regarding the mechanism of the preconditioning 
against stunning noted during the first three occlusions. As mentioned above, Bunch et al. (22) have suggested that it is mediated by adenosine $A_{1}$-receptors.

Third goal: mechanism of the cumulative effect of repetitive ischemia. The third goal of this study was to elucidate the mechanism of the cumulative effect of recurrent ischemic episodes on postischemic contractile dysfunction. We postulated that such a mechanism involves recurrent bouts of oxidative damage as a result of recurrent free radical generation after each reperfusion. Our results support this hypothesis. The spin trapping measurements demonstrate that although free radical generation decreased between the 1st and 5th reperfusion, it was not significantly less after the 10th reperfusion compared with the 1st reperfusion (Figs. 12 and 13). This implies that formation of oxygen-derived free radicals continues unabated with each subsequent episode of ischemia and reflow between the 5th and the 10th occlusions.

However, the mere fact that free radicals are formed does not necessarily signify that they cause additional injury. Our next step, therefore, was to determine the functional significance of these recurrent bursts of free radical generation, namely, whether they result in additional stunning. To this end, we investigated the effect of discontinuing MPG after the fifth reflow (group II). We reasoned that if the free radicals produced during the last 5 occlusion-reperfusion cycles do not cause further dysfunction, MPG given during the first 5 cycles only (group II) should be as protective as MPG given throughout the 10 cycles (group I). Our results show the opposite: when the last five cycles were not "protected" by MPG (group II), the beneficial effects of this drug were completely lost (Fig. 6 ), implying that the oxyradicals formed during the last five reflows did cause severe stunning. We then took this approach one step further; to evaluate specifically the last two occlusionreperfusion cycles, we examined the effect of discontinuing MPG after the eighth reflow (group III). Our finding that in group III the protective effects of MPG were mostly lost implies that a significant part of the oxidative injury responsible for stunning in this model must develop during the 9th and 10th occlusion-reperfusion cycles. (Parenthetically, the fact that significant oxyradical injury continues to develop after the first five occlusion-reperfusion cycles provides further evidence against the presence of a preconditioning effect.)

Taken together, the results of this study indicate that the oxidative stress associated with the last five, or even two, occlusion-reperfusion cycles is sufficient to cause severe myocardial stunning. This finding supports the concept that the cumulative effect of repetitive ischemia on contractility is mediated by the cumulative damage caused by the recurrent bursts of free radical generation associated with each reperfusion.

Relation between collateral flow and postischemic wall thickening. An interesting finding of this study is that the relation between collateral flow and postischemic wall thickening became progressively weaker as the number of ischemic episodes increased. This correlation was close in dogs undergoing one 5-min LAD occlusion (group V; Fig. 11) but much less strong in dogs undergoing three occlusions (group VI; Fig. 11). In dogs undergoing 10 occlusions (group IV), the correlation between postischemic wall thickening and collateral flow during ischemia was reasonably good after the first reperfusion, but then deteriorated progressively and at the end of the 10 occlusion-reperfusion cycles was insignificant (Fig. 8). This finding agrees with a previous report by Cohen and Downey (10), who found a moderately good correlation after the 1st and 2nd reperfusion but no significant correlation after the 12th reperfusion, and by Nicklas et al. (6), who reported no correlation after 105 -min occlusions. The lack of correlation noted after 105 -min occlusions in group IV is in contrast not only with the strong correlation noted after 15 -min occlusion in group $\mathrm{V}$, but also with the correlation observed after 1 15-min occlusion $(2,31,34,51)$.

On the basis of these considerations, we conclude that collateral flow during occlusion is a major determinant of the severity of myocardial stunning after a single ischemic episode but not after multiple ischemic episodes. Other as yet unknown factors must govern the severity of stunning after repetitive ischemia. Regardless of the exact reason(s), this discrepancy further underscores the differences in the pathophysiology of myocardial stunning between the setting of multiple ischemic episodes and the setting of a single ischemic episode, suggesting that findings obtained in one model may not necessarily be applicable to the other.

Clinical implications. Taken together, the results of this study argue strongly against the notion that myocardium exposed to brief ischemia is protected against stunning so that, after five or more occlusion-reperfusion cycles, additional cycles have little effect on recovery of contractility. Instead, our results suggest that recurrent episodes of ischemia continue to cause additional injury to the contractile function of the myocyte and that the intensity of such injury is sufficient to produce severe postischemic dysfunction, even after as many as eight occlusion-reperfusion cycles. Thus, it is theoretically possible that, with repetitive ischemia, myocardial stunning could become protracted or even chronic.

This concept could have important clinical implications. It is now well established that many patients with coronary artery disease develop recurrent brief episodes of ischemia (painful or painless) in the same territory as a consequence of recurrent spasm and/or thrombosis (5). Ambulatory electrocardiographic studies suggest that the frequency of such episodes (often silent) can be quite high, up to $10-20$ times a day $(52,53)$. If applicable to humans, our results suggest that under these circumstances the myocardium might remain reversibly depressed for prolonged periods of time or even chronically. Chronic contractile dysfunction secondary to repetitive stunning has been demonstrated recently in a conscious porcine model of coronary stenosis (54). In the clinical setting, the concept of chronically stunned myocardium would be congruent with a number of observations (for review see reference 11); for example, with the finding that depressed left ventricular function often improves after coronary revascularization with bypass surgery or angioplasty in segments that had normal or near-normal perfusion before revascularization (55-57). In addition, our results imply that antioxidant therapy could effectively attenuate or even prevent the development of such prolonged depression of contractility. This would add a new, intriguing dimension to the oxyradical hypothesis of myocardial stunning. Indeed, if recurrent ischemia causes cumulative loss of function in humans and if oxygen radicals are an important mediator of this phenomenon, then antioxidant therapy would have a considerably greater clinical role. Finally, the present results suggest that MPG may be useful in the prevention of chronic postischemic 
dysfunction. This agent is active orally and thus could be given as a prophylactic therapy to patients at high risk.

\section{Acknowledgments}

We thank Jennifer S. Pocius and Alex Tumang for excellent technical assistance and Valerie R. Price and Joey Manley for expert secretarial assistance.

This study was supported in part by National Institutes of Health R01 grants HL-43151 and HL-55757 (R. Bolli).

\section{References}

1. Heyndrickx, G. R., R. W. Millard, R. J. McRitchie, P. R. Maroko, and S. F. Vatner. 1975. Regional myocardial functional and electrophysiological alterations after brief coronary artery occlusion in conscious dogs. J. Clin. Invest. 56:978985.

2. Bolli, R., W. X. Zhu, J. I. Thomby, P. G. O'Neill, and R. Roberts. 1988. Time-course and determinants of recovery of function after reversible ischemia in conscious dogs. Am. J. Physiol. 254:H102-H114.

3. Braunwald, E., and R. A. Kloner. 1982. The stunned myocardium: prolonged, postischemic ventricular dysfunction. Circulation. 66:1146-1149.

4. Bolli, R. 1992. Myocardial "stunning" in man. Circulation. 86:16711691.

5. Fuster, V., L. Badimon, M. Cohen, J. A. Ambrose, J. J. Badimon, and J. Chesebro. 1988. Insights into the pathogenesis of acute ischemic syndromes. Circulation. 77:1213-1220.

6. Nicklas, J. M., L. C. Becker, and B. H. Bulkley. 1985. Effects of repeated brief coronary occlusion on regional left ventricular function and dimension in dogs. Am. J. Cardiol. 56:473-478.

7. Stahl, L. D., T. R. Aversano, and L. C. Becker. 1986. Selective enhancement of function of stunned myocardium by increased flow. Circulation. 74:843-851.

8. Becker, L. C., J. H. Levine, A. F. DiPaula, T. Guarnieri, and T. R. Aversano. 1986. Reversal of dysfunction in postischemic stunned myocardium by epinephrine and postextrasystolic potentiation. J. Am. Coll. Cardiol. 7:580-589.

9. Stahl, L. D., H. R. Weiss, and L. C. Becker. 1988. Myocardial oxygen consumption, oxygen supply/demand heterogeneity, and microvascular patency in regionally stunned myocardium. Circulation. 77:865-872.

10. Cohen, M. V., and J. M. Downey. 1990. Myocardial stunning in dogs: preconditioning effect and influence of coronary collateral flow. Am. Heart J. 120:282-291.

11. Bolli, R. 1990. Mechanism of myocardial "stunning." Circulation. 82:723-738

12. Gross, G. J., S. T. O'Rourke, L. R. Pelc, and D. C. Warltier. 1992. Myocardial and endothelial dysfunction after multiple, brief coronary occlusions: role of oxygen radicals. Am. J. Physiol. 263:H1703-1709.

13. Halliwell, B., and J. M. C. Gutteridge. 1984. Oxygen toxicity, oxygen radicals, transition metals and disease. Biochem. J. 219:1-14.

14. Hoffmeister, H. M., M. Mauser, and W. Schaper. 1986. Repeated episodes of regional myocardial ischemia: effect on local function and high energy phosphate levels. Basic Res. Cardiol. 81:361-372.

15. Figueras, J., J. Cinca, G. Senador, and J. Rius. 1986. Progressive mechanical impairment associated with progressive but reversible electrocardiographic ischaemic changes during repeated brief coronary artery occlusions in pigs. Cardiovasc. Res. 20:797-806.

16. Warner, K. G., S. F. Khuri, W. Marston, S. Sharma, M. D. Butler, S. N. Assousa, A. J. Saad, Y. Siouffi, and P. T. Lavin. 1989. Significance of the transmural diminution in regional hydrogen ion production after repeated coronary artery occlusions. Circ. Res. 64:616-628.

17. Homan, D. C., D. D. Laxson, E. Sublett, P. Lindstrom, and J. Bache. 1989. Cumulative deterioration of myocardial function after repeated episodes of exercise-induced ischemia. Am. J. Physiol. 256:H1462-H1471.

18. Schott, R. J., S. Rohmann, E. R. Braun, and W. Schaper. 1990. Ischemic preconditioning reduces infarct size in swine myocardium. Circ. Res. 66:11331142.

19. Wynsen, J. C., D. Kenny, H. L. Brooks, and D. C. Warltier. 1991. Regional myocardial function after repetitive brief episodes of ischemia: effect of altering the duration of the reperfusion period. Am. Heart J. 121:1331-1338.

20. Vinten-Johansen, J., P. A. Gayheart, W. E. Johnston, J. S. Julian, and R. A. Cordell. 1991. Regional function, blood flow, and oxygen utilization relations in repetitively occluded-reperfused canine myocardium. Am. J. Physiol. 261:H538-H547.

1. Gross, G. J., S. T. O'Rourke, L. R. Pelc, and D. C. Warltier. 1992 Myocardial and endothelial dysfunction after multiple, brief coronary occlusions: role of oxygen radicals. Am. J. Physiol. 263:H1703-H1709.
22. Bunch, F. T., J. Thornton, M. V. Cohen, and J. M. Downey. 1992. Adenosine is an endogenous protectant against stunning during repetitive ischemic episodes in the heart. Am. Heart J. 124:1440-1446.

23. Weiner, J. M., C. S. Apstein, J. H. Arthur, F. A. Pirzada, and W. B. Hood, Jr. 1976. Persistence of myocardial injury following brief periods of coronary occlusion. Cardiovasc. Res. 10:678-686.

24. Lange, R., J. Ware, and R. A. Kloner. 1984. Absence of a cumulative deterioration of regional function during three repeated 5 or 15 minute coronary occlusions. Circulation. 69:400-408.

25. Yao, Z., and G. J. Gross. 1993. Glibenclamide antagonizes adenosine A receptor-mediated cardioprotection in stunned canine myocardium. Circulation. 88:235-244.

26. Bolli, R., B. S. Patel, W. X. Zhu, P. G. O'Neil, M. L. Charlat, and R. Roberts. 1987. The iron chelator desferrioxamine attenuates postischemic ventricular dysfunction. Am. J. Physiol. 253:H1372-H1380.

27. Bolli, R., B. S. Patel, M. O. Jeroudi, E. K. Lai, and P. B. McCay. 1988 Demonstration of free radical generation in "stunned" myocardium of intact dogs with the use of the spin trap $\alpha$-phenyl $N$-tert-butyl nitrone. J. Clin. Invest. 82:476485.

28. Bolli, R., M. O. Jeroudi, B. S. Patel, C. M. DuBose, E. K. Lai, R. Roberts, and P. B. McCay. 1989. Direct evidence that oxygen-derived free radicals contribute to postischemic myocardial dysfunction in the intact dog. Proc. Natl. Acad. Sci. USA. 86:4695-4699.

29. Bolli, R., M. O. Jeroudi, B. S. Patel, O. I. Aruoma, B. Halliwell, E. K Lai, and P. B. McCay. 1989. Marked reduction of free radical generation and contractile dysfunction by antioxidant therapy begun at the time of reperfusion evidence that myocardial "stunning" is a manifestation of reperfusion injury. Circ. Res. 65:607-622.

30. Bolli, R., B. S. Patel, M. O. Jeroudi, X. Y. Li, J. F. Triana, E. K. Lai, and P. B. McCay. 1990. Iron-mediated radical reactions upon reperfusion contribute to myocardial "stunning." Am. J. Physiol. 259:H1901-H1911.

31. Triana, J. F., X. Y. Li, U. Jamaluddin, J. I. Thornby, and R. Bolli. 1991. Postischemic myocardial "stunning": identification of major differences between the open-chest and the conscious dog and evaluation of the oxygen radical hypothesis in the conscious dog. Circ. Res. 69:731-747.

32. Li, X. Y., P. B. McCay, M. Zughaib, M. O. Jeroudi, J. F. Triana, and R. Bolli. 1993. Demonstration of free radical generation in the "stunned" myocardium in the conscious dog and identification of major differences between conscious and open-chest dogs. J. Clin. Invest. 92:1025-1041.

33. Sun, J. Z., H. Kaur, B. Halliwell, X. Y. Li, and R. Bolli. 1993. Use of aromatic hydroxylation of phenylalanine to measure production of hydroxyl radicals after myocardial ischemia in vivo: direct evidence for a pathogenetic role of the hydroxyl radical in myocardial stunning. Circ. Res. 73:534-549.

34. Sekili, S., P. B. McCay, X. Y. Li, M. Zughaib, J. Z. Sun, X. L. Tang, J. I. Thornby, and R. Bolli. 1993. Direct evidence that the hydroxyl radical plays a pathogenetic role in myocardial "stunning" in the conscious dog and that stunning can be markedly attenuated without subsequent adverse effects. Circ. Res. 73:705-723.

35. Devi, P. U. 1983. Chemical radiation protection by alpha-mercaptopropionyl glycine. J. Nucl. Med. Allied Sci. 27:327-336.

36. Sedlak, J., and R. H. Lindsay. 1968. Estimation of total, protein-bound, and nonprotein sulfhydryl groups in tissue with Ellman's reagent. Anal. Biochem. 25:192-205.

37. Bolli, R., and P. B. McCay. 1990. Use of spin traps in intact animals undergoing myocardial ischemia/reperfusion: a new approach to assessing the role of oxygen radicals in myocardial "stunning." Free Radical Research Communications. 9:169-180.

38. Wallenstein, S., C. L. Zucker, and J. L. Fleiss. 1980. Some statistical methods useful in circulation research. Circ. Res. 47:1-9.

39. SAS Institute. 1988. SAS/STAT User's Guide, Release 6.03 Edition. SAS Institute, Cary, NC. 19-26, 125-154, 713-726.

40. Bolli, R., J. F. Triana, and M. O. Jeroudi. 1990. Prolonged impairment of coronary vasodilation after reversible ischemia: evidence for microvascular "stunning." Circ. Res. 67:332-343.

41. McCay, P. B. 1985. Vitamin E: interactions with free radicals and ascorbate. Annu. Rev. Nutr. 5:323-340.

42. McCay, P. B., E. K. Lai, J. L. Poyer, C. M. DuBose, and E. G. Janzen. 1984. Oxygen-and carbon-centered free radical formation during carbon tetrachloride metabolism. J. Biol. Chem. 259:2135-2142.

43. Murry, C. E., V. J. Richard, R. B. Jennings, and K. A. Reimer. 1989. Free radicals do not cause myocardial stunning after four 5 minute coronary occlusions. Circulation. 80(Suppl. II):II-296. (Abstr.)

44. Rohmann, S., R. J. Schott, E. Braun, and W. Schaper. 1990. SOD and catalase do not prevent stunning or effect ischemic preconditioning in swine myocardium. J. Mol. Cell. Cardiol. 22:S48. (Abstr.)

45. Reimer, K. A., C. E. Murry, I. Yamasawa, M. L. Hill, and R. B. Jennings. 1986. Four brief periods of myocardial ischemia cause no cumulative ATP loss or necrosis. Am. J. Physiol. 251:H1306-H1315. 
46. Geft, I. L., M. C. Fishbein, K. Ninomiya, J. Hashida, E. Chaux, J. Yano, J. Y-Rit, T. Genov, W. Shell, and W. Ganz. 1982. Intermittent brief periods of ischemia have a cumulative effect and may cause myocardial necrosis. Circulation. 66:1150-1153.

47. Basuk, W. H. O., K. A. Reimer, and R. B. Jennings. 1986. Effect of repetitive brief episodes of ischemia on cell volume, electrolytes and ultrastructure. J. Am. Coll. Cardiol. 8:33A-41A.

48. Henrichs, K. J., H. Matsuoka, and J. Schaper. 1987. Influence of repetitive coronary occlusions on myocardial adenine nucleosides, high energy phosphates and ultrastructure. Basic Res. Cardiol. 82:557-565.

49. Murry, C. E., R. B. Jennings, and K. A. Reimer. 1986. Preconditioning with ischemia: a delay of lethal cell injury in ischemic myocardium. Circulation. 74:1124-1136

50. Downey, J. M. 1992. Ischemic preconditioning: nature's own cardioprotective intervention. Trends Cardiovasc. Med. 2:170-176.

51. Gross, G. J., N. E. Farber, H. F. Hardman, and D. C. Warltier. 1986. Beneficial actions of superoxide dismutase and catalase in stunned myocardium of dogs. Am. J. Physiol. 250:H372-H377.

52. Deanfield, J. E., A. P. Selwyn, S. Chierchia, A. Maseri, P. Ribeiro, S.
Krikler, and M. Morgan. 1983. Myocardial ischaemia during daily life in patients with stable angina: its relation to symptoms and heart rate changes. Lancet. 2:753758

53. Cohn, P. F. 1985. Silent myocardial ischemia: classification, prevalence, and prognosis. Am. J. Med. 79(Suppl. 3A):2-12.

54. Shen, Y. T., N. Hasebe, H. Zhang, and S. F. Vatner. 1993. Impaired regional myocardial function during ameroid-induced chronic coronary artery stenosis in conscious pigs: hibernation or stunning? Circulation. 88:1-188. (Abstr.)

55. Tillisch, J., R. Brunken, R. Marshall, M. Schwaiger, M. Mandelkern, M. Phelps, and H. Schelbert. 1986. Reversibility of cardiac wall-motion abnormalities predicted by positron-tomography. $N$. Engl. J. Med. 314:884-888.

56. Nienaber, C. A., R. C. Brunken, C. T. Sherman, L. A. Yeatman, S. S Gambhir, J. Krivokapich, L. L. Demer, O. Ratib, J. S. Child, M. E. Phelps, and H. R. Schelbert. 1991. Metabolic and functional recovery of ischemic human myocardium after coronary angioplasty. J. Am. Coll. Cardiol. 18:966-978.

57. Vanoverschelde, J. L. J., W. Wijns, C. Depré, B. Essamri, G. R. Heyndrickx, M. Borgers, A. Bol, and J. A. Melin. 1993. Mechanisms of chronic regiona postischemic dysfunction in humans. New insights from the study of noninfarcted collateral-dependent myocardium. Circulation. 87:1513-1523. 